\title{
Soft coral and sea fan (Octocorallia) biodiversity and distribution from a multi- taxon survey (2009-2014) of the shallow tropical Kimberley, Western Australia
}

\author{
Monika Bryce $^{{ }^{*}}$, Ben Radford ${ }^{2}$ and Katharina Fabricius ${ }^{3}$ \\ 1 Department of Aquatic Zoology, Western Australian Museum, Locked Bag 49, Welshpool DC, \\ Western Australia 6986, Australia. \\ ${ }^{2}$ Australian Institute of Marine Science, 35 Stirling Hwy, Crawley, Western Australia 6009, Australia. \\ ${ }^{3}$ Australian Institute of Marine Science, 1526 Cape Cleveland Road, Cape Cleveland, Queensland \\ 4810, Australia. \\ * Email: monika.bryce@museum.wa.gov.au
}

\begin{abstract}
Any assessment of tropical reef ecosystems should extend beyond a hard coral inventory to include soft corals and other sessile benthic organisms. We assessed octocoral assemblages at 177 marine survey stations in the Kimberly region, Western Australia ( $<30 \mathrm{~m}$ depth), to establish species inventories and describe community structures. The 1,174 vouchered specimens yielded a total of 206 Linnean and morphospecies. The overall composition of this fauna was typical for tropical Indo-Pacific shallow water coral reefs. Octocoral communities demonstrated high species heterogeneity across habitats, with strong differences between inshore and offshore, and a latitudinal zonation along the Kimberley inshore. Of the recorded taxa, 45\% were exclusively found offshore, $16 \%$ inshore and $39 \%$ occurred both inshore and offshore. The nearshore Bonaparte Peninsula, the midshelf region and the northern offshore regions were all 'hotspots' for soft coral diversity, while the southern offshore regions presented the most distinct species composition. These results have profound implications for conservation strategies relating to these remote benthic habitats.
\end{abstract}

KEYWORDS: Indian Ocean, Kimberley Marine Bioregion, Octocorals, Alcyonacea, Coelenterata, Cnidaria, ecological assessment, Woodside Collection Project

\section{INTRODUCTION}

The marine environments of the Kimberley, Western Australia (WA) are currently of significant interest as they are one of the last remaining large, unspoilt regions on Earth with high conservation values and a growing nature based economy (Department of Parks and Wildlife 2016). This conflicts with proposed industrial development associated with large oil and gas reserves (Department of Environment and Conservation 2009).

The importance of utilising natural history collections to provide baseline biodiversity information to inform conservation and environmental management decisions has long being recognised, but baseline data suitable for 'characterising the assets' in this region are scarce (Hooper and Ekins 2004; Blakeway and Radford 2004; Wood and Mills 2008; Pyke and Ehrlich 2010). In particular, soft corals and sea fans (Octocorallia; Order Alcyonacea) have received little collecting effort in WA waters, and even less in the Kimberly, resulting in a scarcity of publications and curated collections (Kükenthal 1910; Broch 1916; Marsh 1986, 1992, 1993; Griffith 1997; Keesing et al. 2011; Bryce and Sampey 2014). This study represents the first SCUBA collections from the Kimberley in recent times (2009 to 2014).

The Woodside Collection Project (Kimberley) was a multi-taxon marine biodiversity survey program. It completed 181 survey stations (Bryce et al. 2018) within a specified Project Area covering 476,000 $\mathrm{km}^{2}$ (Sampey et al. 2014), which extended from the 
Kimberley coast to the shelf edge. Here we report on the octocoral results for 181 survey stations, and over 1000 vouchered specimens. Furthermore, we discuss octocoral biodiversity, and implications for the marine habitat classification of the region.

Despite the diversity of organisms recognised as corals, benthic studies of tropical reefs are dominated by hard corals, thus a decline in coral cover is largely defined by declines of scleractinian corals (Przeslawski et al. 2008; Edmunds et al. 2014). The importance of hard corals in the formation of significant reef structures is well known, but future global warming trends point to a decline in their dominance (Blakeway and Radford 2004; Hoegh-Guldberg et al. 2007; Hughes et al. 2010; Edmunds et al. 2014). However, octocorals also play a large role in reef communities, and may become a dominant feature of future reef systems following a decline of hard corals (Przeslawski 2008; Smith et al. 2016; Baum et. al. 2016). Transitions to octocoral dominance have been reported from the IndoPacific following environmental disturbances of coral reef systems, for example caused by bleaching events, blast fishing, coral predation by crown of thorns seastars, or decline in water quality (Benayahu and Loya 1981; Bradbury and Mundy 1989; Fabricius 1996, 1997, 1998; Fox et al. 2003; Ruzicka et al. 2013; Lenz et al. 2015; Smith et al. 2016; Baum et. al. 2016).

Soft corals and sea fans occur worldwide in all reef habitats from the intertidal to abyssal depths. In the tropical Indo-Pacific they are represented by 90 genera and 23 families (Fabricius and Alderslade 2001). They are a major component of coral reef communities and marine benthos, and among the most important contributors to the total biomass of Indo-Pacific coral reef systems; they may cover up to $25 \%$ of the total reef substratum (Tursch and Tursch 1982; Fabricius and Alderslade 2001). Although most octocorals contribute little to the formation of limestone reef structure, members of the alcyoniid genus, Sinularia, are active reef builders depositing large amounts of structural calcareous sclerites into the rock layers (Jeng et al. 2011). Despite their ecological importance as filter feeders, the diversity of octocorals remains poorly known and new species, genera and families are still discovered and described on a regular basis (e.g. Breedy et al. 2012; McFadden and van Ofwegen, 2013; Bryce and Poliseno 2014; Bryce et al. 2015). Surveys into octocoral diversity are fundamental to assessing the biodiversity of ecosystems with respect to ecosystem function (food web dynamics and conductivity), assessing the long term changes in coral reef community structure and understanding and monitoring the outcomes of habitat restoration activities (Williams 1992, Benayahu et al. 2003, Fabricius et al. 2007, Chanmethakul et al. 2010, Benayahu et al. 2012, Shackleton and Rees 2016).

\section{AIMS}

The aim of this study was to record the biodiversity of the shallow water $(<30 \mathrm{~m})$ soft coral and gorgonian fauna in the Kimberley region of WA over a wide range of habitats and locations, and to comment on diversity trends, community composition and spatial patterns of this faunal group.

\section{METHODS}

\section{STUDY AREA AND SITE DESCRIPTIONS}

Octocoral vouchers were collected as part of a Western Australian Museum (WAM) series of biodiversity surveys in the Kimberley (2009-2014). Stations were within the Project Area defined by Sampey et al. (2014), in an area westward from the coast, including several midshelf sites (Browse Island, Eugene McDermott, Echuca, Heywood and Vulcan Shoals), to the continental edge atolls, from Rowley Shoals in the south to Ashmore and Hibernia Reefs in the north. Inshore stations covered reefs and islands near Cape Leveque in the south to Long Reef in the north (Figure 1). Bryce et al. (2018) provide a full explanation and description of the study area, including coordinates and general station data. Collections were made intertidally and by SCUBA, at depths ranging from $0-30 \mathrm{~m}$. (Figures 1 and 2).

Distinct differences in environmental conditions and connectivity regimes have been recorded from the coast to the shelf edge (Wilson 2013, 2014). The Kimberley inshore (shoreline to $50 \mathrm{~m}$ bathymetry as defined by Bryce et al. 2018) is characterised by relatively turbid, macrotidal, nutrient rich conditions, with connectivity regimes controlled by local currents influenced by seasonal winds and tidal flows (Figure 2A, C). Within the midshelf (51-150 m bathymetry) are submerged shoals (Vulcan, Eugene McDermott, Heywood and Echuca Shoals) with a hard rock foundation and low coral cover and Browse Island (Heyward et al. 2012, Wilson 2014).The offshore area (>150 m bathymetry) includes Ashmore and Hibernia Reefs in the north and Rowley Shoals in the south, all situated on the edge of the continental shelf approximately 300 $\mathrm{km}$ off the coast (Figure 1). They are characterised by clear, nutrient poor, oceanic conditions, with direct pelagic connectivity to the highly biodiverse 


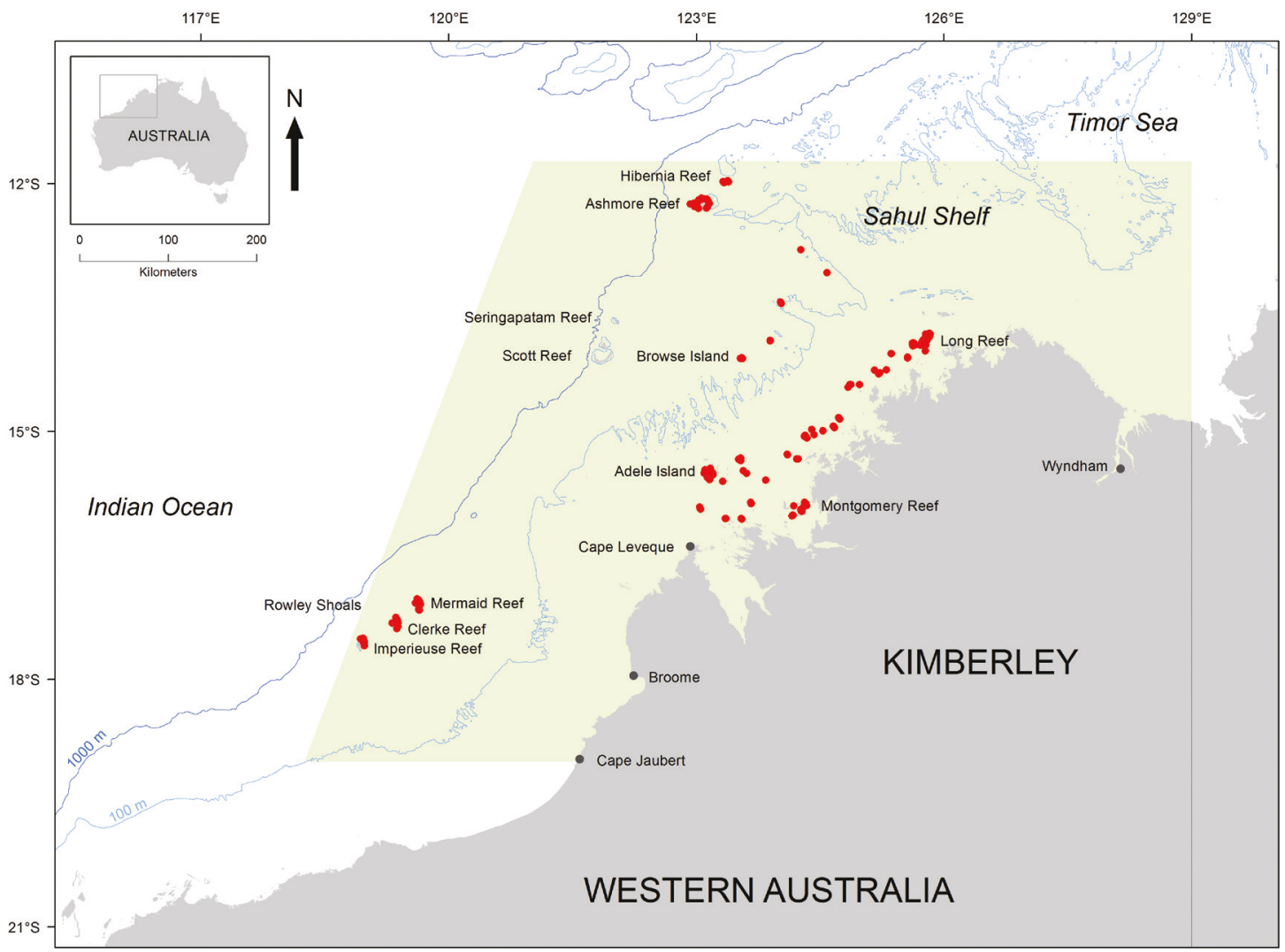

FIGURE $1 \quad$ Location of octocoral survey stations (red dots) in the Project Area (shaded green; 476,000 km2). The Project Area was divided into continental shelf zones, using the following bathymetric ranges: inshore (coastal shoreline to $50 \mathrm{~m}$ depth including all inshore islands and reefs), midshelf $(51-150 \mathrm{~m}$ ) and offshore (>150 m)

region of Indonesia (Figure 2B, D). Ashmore Reef is the largest and most mature platform reef on the north-western side of the Sahul Shelf $\left(150 \mathrm{~km}^{2}\right)$, and consists of three low vegetated supratidal cays, two shallow lagoons open to the leeward northern side, and an extensive shoal area less than $50 \mathrm{~m}$ deep. The southern and windward reef edge has an unbroken margin backed by extensive sand flats and an outer reef slope dominated by strong surge channels, which develop into spur and groove formations (Wilson 2013). To the north-east of Ashmore Reef is an extensive and complex system of submerged reefs, with only Hibernia Reef being emergent. Hibernia Reef is a coral rich, intertidal platform with a shallow lagoon.

The Rowley Shoals are comprised of three distinct reefs, Imperieuse, Clerke and Mermaid Reefs, separated by deep water. Imperieuse and Clerke Reefs have unvegetated, supratidal sand cays, called Cunningham and Bedwell Islands respectively. The sand cay on Mermaid Reef is intertidal. The oval-shaped atolls have a northsouth aspect and central lagoons. Each atoll has a narrow channel on the north-eastern side and steep drop offs on the western sides.

\section{TAXONOMIC AND COLLECTION SCOPE}

Percentage cover: Point intercept data collection was undertaken at 164 of 181 stations at both subtidal and intertidal sites. Of the midshelf sites, point intercept transects were only undertaken at Browse Island as all other midshelf stations were deep depths. The data was analysed for percent habitat cover for biotic (Octocorallia, Hexacorallia, Porifera, other invertebrates, seagrass, turf algae, macroalgae, crustose algae and coralline algae) and abiotic (sand, rubble and shell-grit, rock and silt) functional groups (See Richards et al. 2018 for a full explanation).

Biodiversity surveys: Octocoral biodiversity surveys were conducted at 177 of 181 survey stations. Dives at four stations (16/K09, 70/K11, $71 / \mathrm{K} 11$ and $92 / \mathrm{K} 12$ ) were abandoned due to poor dive conditions. Transects were swum at 153 stations (nine stations had no species records and 15 had only biodiversity information). For these stations, soft corals were surveyed along two 50x2 $\mathrm{m}$ wide belt transects (T1, T2) utilising transect tape as a guide. Transect depth was between 12 and $15 \mathrm{~m}$, independent of the tide due to the 

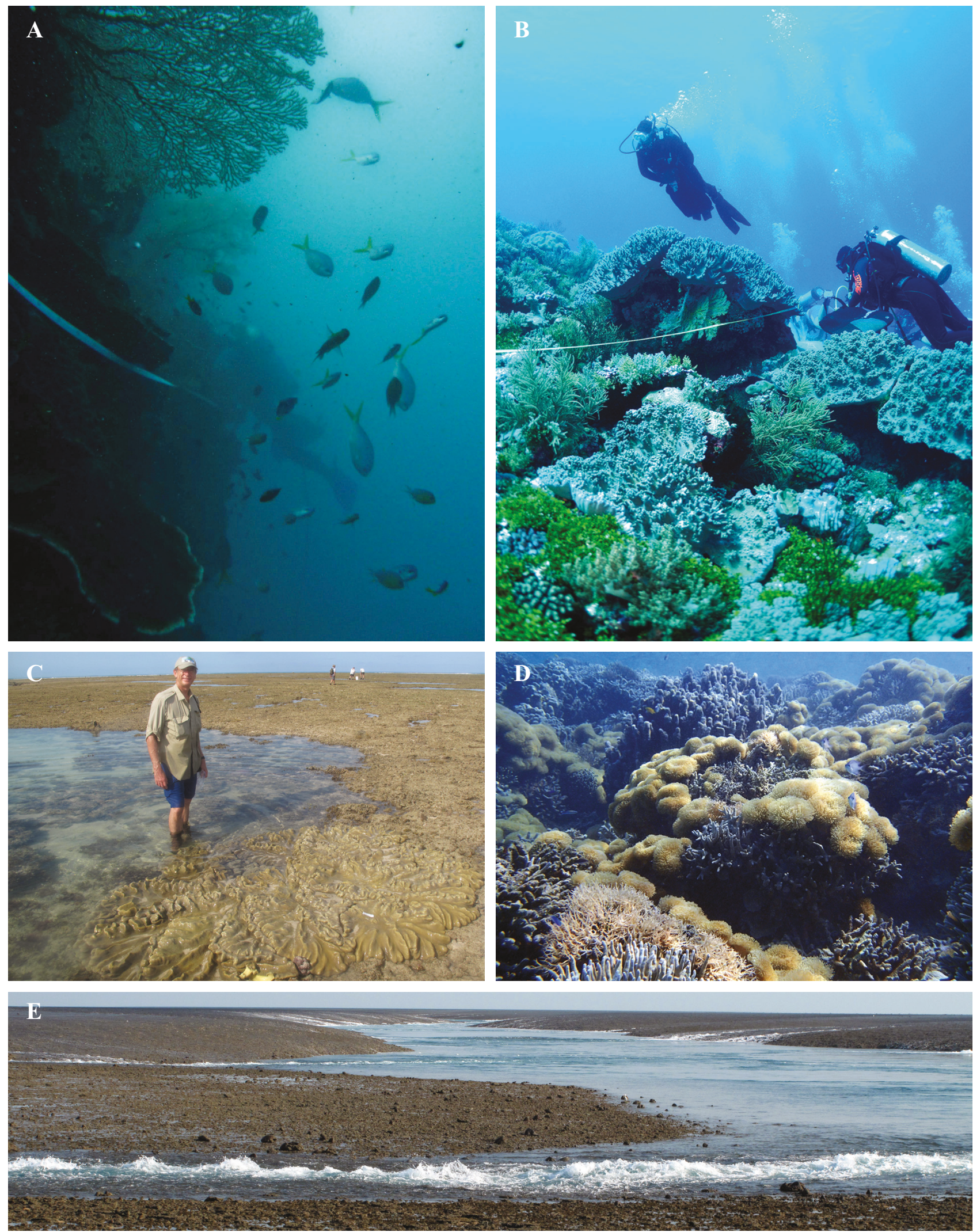

FIGURE 2 Examples of habitats: A) Cassini Island (station 30/K10). Turbid conditions in the inshore subtidal. Sea fans indicate a periodic, strong current flow; B) Hibernia Reef (station 144/K113). Clear conditions on a fore-reef slope in the offshore subtidal. Large areas are covered by a variety of octocorals; C) White Island (station $66 / K 11)$. A massive Lobophytum specimen on an exposed intertidal mid-littoral platform in the inshore area; D) Ashmore Reef (station 138/K13). A large mid-littoral tide pool with soft corals (Sarcophyton) in the offshore; E) Montgomery Reef (station 51/K09). Inshore habitat with several fast flowing water channels bisecting the site on a falling tide indicating a high energy, intertidal zone. 
prevailing macrotidal regime of the region (see Bryce et al. 2018). All octocorals encountered within the survey area were identified and abundance recorded. Species abundances for each transect $(\mathrm{T} 1, \mathrm{~T} 2)$ were counted to a maximum of 20 individuals. Station effort was standardised at 60 minutes and any post-transect survey time up to the 60 minute threshold was spent identifying, recording (presence/absence data only) and vouchering off-transect octocoral species. Octocoral voucher specimens were collected at each station for further taxonomic determinations to species level (either Linnean species or OTUs (Operational Taxonomic Units). The concept of morphospecies assumes that each OTU represents a single species, which has not been identified within the Linnaean system; they have differences in morphological characters from published descriptions and/or are a preliminary identification. Subsequent research will determine if they represent an undescribed species or have been previously described in historic taxonomic literature. Matching OTU data within collections can create large datasets that can significantly enhance our understanding of biodiversity, ecology and distributional trends. This approach has proven useful for difficult sessile invertebrate determinations (Fromont et al. 2016). All specimens have been registered and deposited in the WAM, Perth.

\section{MORPHOLOGICAL IDENTIFICATION}

Specimens were photographed in situ and on deck, and then preserved in $75 \%$ ethanol with subsamples for DNA analysis preserved in absolute ethanol (100\%). Sclerites were prepared for microscopy by cutting small pieces of the specimen from five different regions (polyps, surface of the polyp region, surface of the base, interior of the polyp region, interior of the base) and dissolving them in sodium hypochlorite $(13 \%$ available chlorine). After the organic material had dissolved, the sclerites were rinsed with distilled water and dried on a glass microscope slide for further investigation. Durcupan ACM was used as a mounting medium for permanent slides (Fabricius and Alderslade 2001: 40).

\section{SPATIAL INFORMATION, COLLECTION DETAILS AND MAPPING}

Location and collecting details were checked and verified, and the location of the specimen records visualised using ESRI ArcGIS 10.4.1. Maps of species richness and sampling effort were generated for each main location. The full list of locations, latitude and longitude and other relevant collection information for all taxa is in Bryce et al. (2018), Table 1.

\section{STATISTICAL ANALYSES}

Multivariate analyses of transect cover data were performed in the software package PRIMER v6 (Clarke and Warwick 2001; Clarke and Gorley 2006). Analyses were based on square root transformed habitat data per station using a Bray Curtis similarity coefficient and Non metric Multidimensional Scaling (MDS). ANOSIM was conducted on the factors, continental shelf zones (inshore/offshore) and tidal zones (subtidal/ intertidal) to test for significant differences in benthic community structure.

Diversity was based on presence/absence data and calculated from the species inventory (number of species at site; $\mathrm{R}$ version 3.2.2. data analyses). Alpha diversity was completed for the 177 octocoral survey stations and separately for the 153 transect stations.

For species composition analyses a hierarchical cluster analysis (Ward's method) was conducted based on species presence/absence data for each transect site to group sites and define spatial patterns (De Cáceres and Legendre 2009; De Cáceres et al. 2010; Dufrêne and Legendre 1997). Original clusters were generated using a Bray Curtis dissimilarity analysis of a binary species presence/absence matrix for the 153 biodiversity transects examined. A matrix dendrogram was used to aggregate soft coral stations into groups based on composition similarity and groups were plotted onto a map to visualise spatial patterns. An indicator species analysis was conducted to determine which species typify each group using the package 'indicspecies' and function multipatt in the statistical software $\mathrm{R}$ version 3.3.1.

\section{RESULTS}

\section{COLLECTING EFFORT AND SPECIES COMPOSITION OF HIGHER OCTOCORAL TAXA}

One hundred and seventy seven stations were surveyed for octocorals. Nine stations had no octocorals present (stations 8, 11 and 25/K09; 73, 82, 84 and 87/K11; 123 and137/K13). One thousand, one hundred and seventy four (1174) specimen lots were collected from the 168 stations where octocorals were present. Transects were swum at 153 stations, while 15 stations had biodiversity only assessments 
due to the absence of octocorals on the transects (stations 72, 89 and 90/K11; 91, 104 and 120/K12) or time limits at deeper depths (stations 107-109 and 121/K12; 146-148/K13; 176 and 181/K14).

Within the subclass Octocorallia, 206 species were from the order Alcyonacea, and one species, Heliopora coerulea (Pallas, 1766) from the order Helioporacea. Within the Alcyonacea all five informal suborders were present, representing 18 families and 59 genera (Bayer 1981) (Table 1). The five subordinal groups comprised 9 Stolonifera octocoral species, 116 Alcyoniina, 27 Scleraxonia, 42 Holaxonia, and 16 Calcaxonia (Figure 3A). Four taxa were identified to genus only. Species belonging to Sinularia (Alcyonacea), Chromonephthya and Dendronephthya (Nephtheidae) were identified to genus only due to high taxonomic uncertainty as a result of a high level of intracolony and intraspecies variability (McFadden et al. 2009). Nevertheless, the high variability in colony shape and colour, and also sclerite size and shape, suggests a high diversity within these genera. Species within Xenia were also identified to genus only due to identification problems (Janes and Mary 2012; McFadden et al. 2017). The following families had the highest number of identified taxa: Alcyoniidae (54), Nephtheidae (33), Plexauridae (33), Melithaeidae (20), Nidaliidae (15) and Ellisellidae (14) (Figure 3B). All other families were represented by only a few species (1-10).

\section{OCTOCORAL COVER AND ZONATION PATTERNS}

Transect point intercept analyses (ANOSIM) demonstrated strong clustering of communities at the functional group level associated with intertidal and subtidal habitats $(\mathrm{R}=0.276, \mathrm{p}=0.0001)$, as well as offshore and inshore localities $(\mathrm{R}=0.415, \mathrm{p}=$ 0.0001) (Figure $4 \mathrm{~A}, \mathrm{C}$ ). Mean octocoral cover varied between $0-30 \%$ (Figure 4B, D). Forty-six percent of stations had extremely low cover or no octocorals $(<1 \%)$, while $48 \%$ had between $1-10 \%$ cover, and seven stations had $>10 \%$ cover. In the intertidal, vectors demonstrated strong habitat affinities of soft corals with coralline algae in the inshore areas and with turf algae offshore, and an overall negative association with rock and silt. In the subtidal, vectors demonstrated strong affinities of soft corals with hard coral and sponge cover inshore and crustose and coralline algae cover offshore, and an overall negative association with macroalgae, turf algae and seagrass. Superimposing octocoral station cover over each station demonstrated low octocoral cover $(0-7 \%)$ for intertidal areas (Figure $4 \mathrm{~B}$ ) and higher octocoral cover (up to $30 \%$ ) at subtidal stations (Figure 4D). Octocoral cover was highest at the offshore subtidal sites of Ashmore and Hibernia Reefs, with the highest cover of 30\% recorded from Hibernia Reef (station 144/K13). From the inshore subtidal stations, Cassini Island (station 34/K10) had the highest cover with $26 \%$.
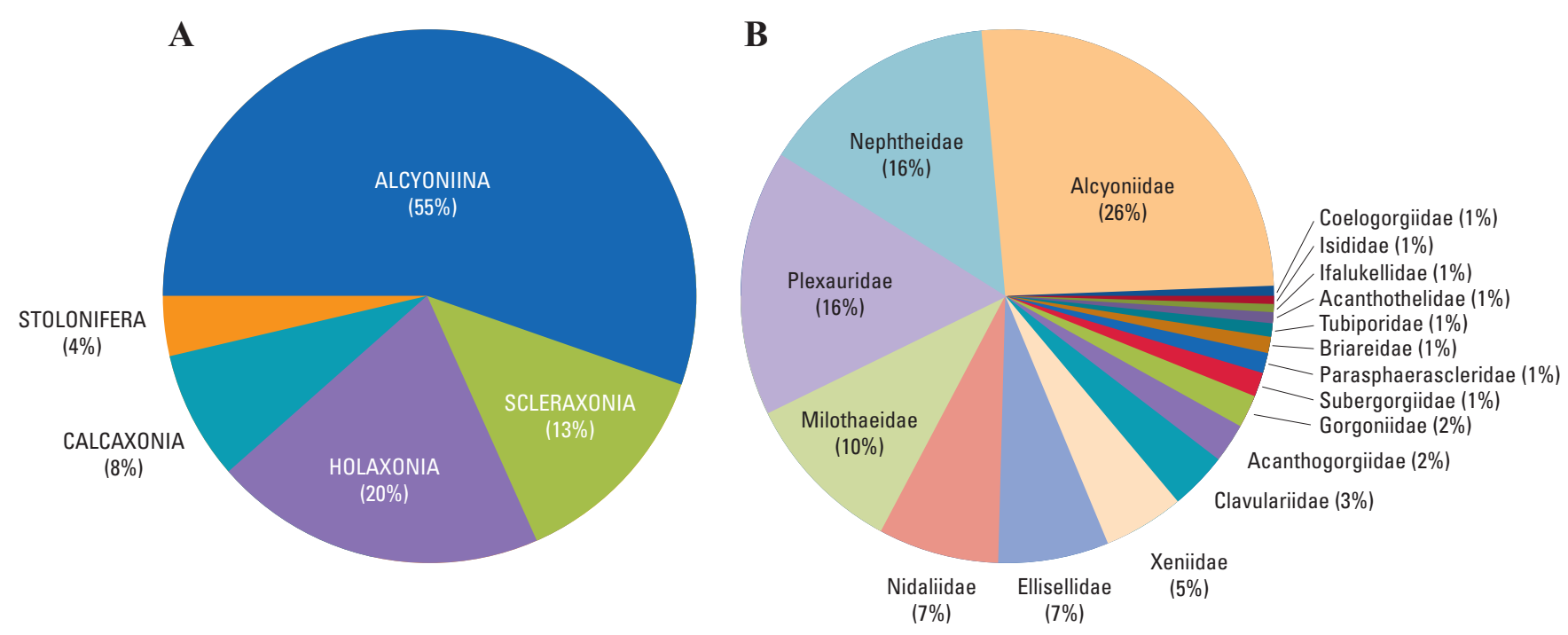

FIGURE 3 Percent composition of octocoral taxa comprising the 206 identified species in the surveys: A) octocoral subordinal groups; B) percentages of identified octocoral taxa within Alcyonacea families (rounded to the nearest percentage). 

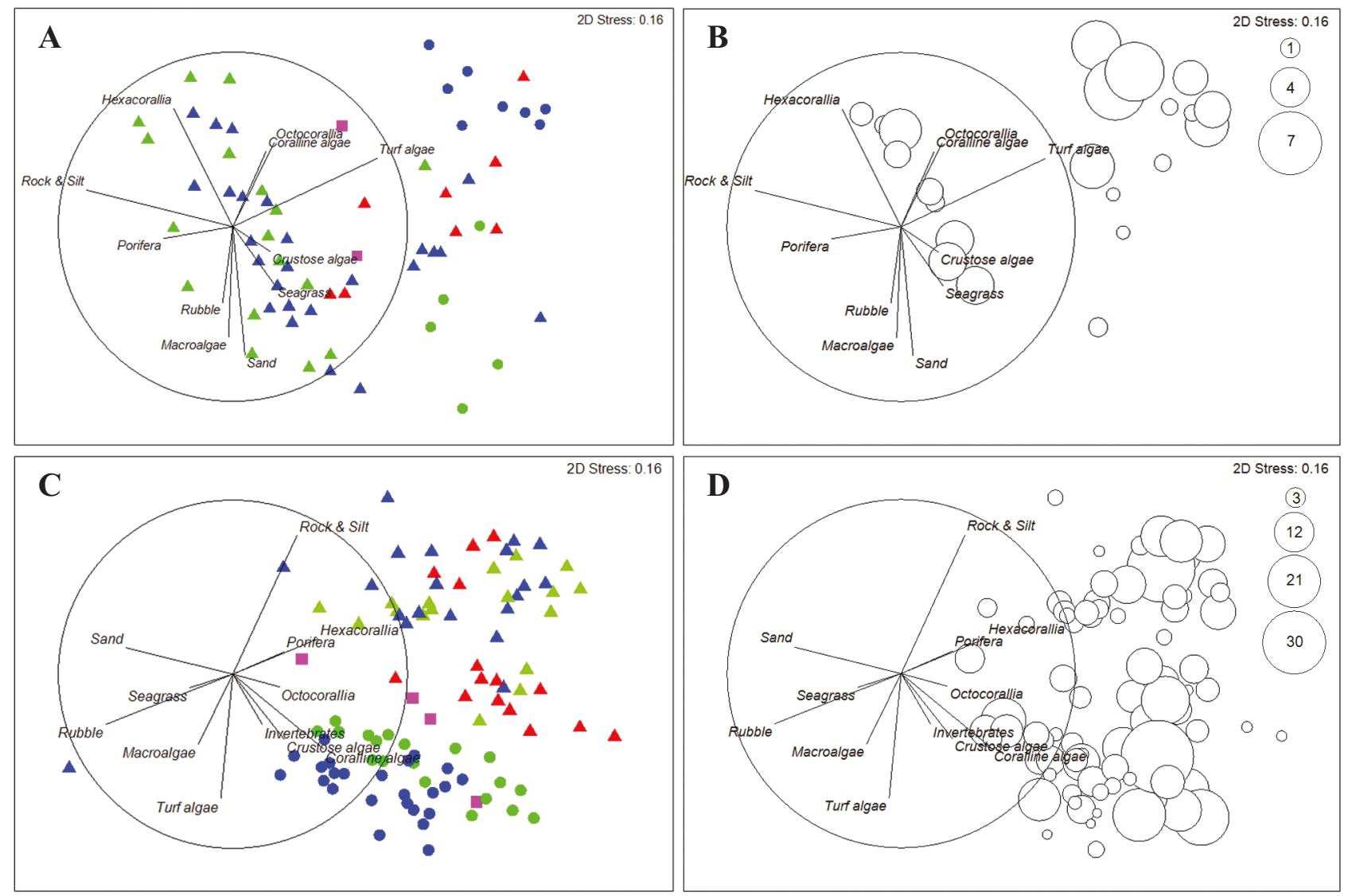

FIGURE $4 \quad$ Non-metric multidimensional scaling ordination plots of transect point intercept data, based on Bray Curtis similarities: Blue triangle: Inshore South; Red triangle: Inshore Central; Green triangle: Inshore North; Pink square: Midshelf; Blue circle: Offshore South; Green circle: Offshore North. A) intertidal MDS plot of habitat cover based on functional groups; B) mean percentage intertidal octocoral cover superimposed over each station; C) subtidal MDS plot of habitat cover based on functional groups; D) mean percentage subtidal octocoral cover superimposed over each station. The vectors indicate the principle drivers of similarity between stations.

\section{SPECIES ASSEMBLAGES}

Octocoral species occurrence varied with locality, especially with respect to offshore versus inshore areas (Table 1). Forty-five percent of species were exclusive to offshore stations, including the midshelf, while $16 \%$ were associated with inshore. Thirty-nine percent occurred at both inshore and offshore areas. Of the specimens identified to genus level, Sinularia, Dendronephthya and Xenia were highly abundant at both inshore and offshore stations and Chromonephthea was only found inshore. Only a few species were relatively evenly distributed throughout the survey area (Table 1). Relative abundances were similar for both offshore and inshore areas (rating scale of 0-5: $0=$ absent; $1=$ 'singletons', one or few colonies (1-3); $2=$ rare (4-10); 3 = uncommon (11-20); 4 = common (21-100); and $5=$ very common $(>101)$ (Fabricius and Déath 2001). Offshore, $40 \%$ of soft corals were represented by singletons, $17 \%$ were rare, $10 \%$ uncommon, $25 \%$ common, and $8 \%$ very common. Similarly, inshore $40 \%$ of soft corals were represented by singletons, $14 \%$ were rare, $13 \%$ uncommon, $22 \%$ common and $11 \%$ very common (Table 1 ).

Within the subordinal group Stolonifera, Coelogorgia palmosa Milne-Edwards and Haime, 1857 (Coelogorgiidae) was a new Australian geographical record from this survey (Figure 5A) (Bryce and Poliseno 2014). C. palmosa was collected from six Ashmore Reef stations and four midshelf shoal stations, and absent from the remaining 18 Ashmore and Hibernia Reef stations on the outer shelf, and all inshore stations. While its abundance at some Ashmore Reef stations was remarkably high, with up to 100 colonies per dive, midshelf abundance was low.

The subordinal group Alcyoniina was very diverse with the families Alcyoniidae (7 genera), Nephtheidae (9), Parasphaerascleridae (1), Nidaliidae (3) and Xeniidae (6) all represented. One 
new species, Parasphaerasclera kimberleyensis Bryce et al. (2015) was found at Long Reef (station 44/ K10). A second species from the newly erected genus Parasphaerasclera, P. grayi (Thomson and Dean, 1931), was collected inshore close to White Island (station 69/K11) and offshore at the Rowley Shoals (station 161/K14) respectively (Figure 5B). $P$. grayi has a wide Indo-Pacific distribution and represents a new record for Australia. These small digitate species were found in shallow water beneath overhangs. Another digitate species, Paraminabea aldersladei, Williams 1992 has also adapted to a cryptic lifestyle, preferring light limited overhangs and caves. It was also found in the inshore Kimberley, but was more numerous in the clear, offshore habitats of the Rowley Shoals, Ashmore and Hibernia Reefs.

The genera Chironephthya and Siphonogorgia (family Nidaliidae) are each represented by seven species (Figure 5C). These genera are typically distinguished by their calyx structure, polyp distribution on the colony, and colony shape. The history of these species is confused, but there is a high likelihood that the Kimberley material incorporates new species (Fabricius and Alderslade 2001; López-González et al. 2014). One specimen, Nephthyigorgia kükenthali Broch 1916, was collected from Rosella Shoals (station 91/ K12), a depauperate, high energy sediment plain of coarse coral fragments and shell grit. This species was first described from Cape Jaubert, south of Broome and thought rare, but is now considered abundant in sandy environments of the Pilbara region (Keesing et al. 2011). This abundance is seen in recent survey material from the Pilbara, undertaken by the Commonwealth Scientific Research Organisation (CSIRO), and from the Kimberley by the Western Australian Marine Science Institute (WAMSI), with identifications by the senior author. These surveys are currently unpublished.

Species of the family Xeniidae are more abundant inshore, but can be considered common from the coast to the outer shelf (Table 1). However, members of the genus Heteroxenia occur mainly on the outer shelf. A specimen (WAM Z67415) of Asterospicularia randalli, with star-like sclerites collected in Mermaid Reef lagoon represents a range extension for WA (Figure 5D). The genus was established in 1951 by Utinomi for a single Taiwan species, $A$. laurae, and later a second species, $A$. randalli was established by Gawel (1976) from Guam. Alderslade (2001) reported the latter in great abundance from the Great Barrier Reef and notes that both species seem closely related or perhaps identical. To clarify the species, $A$. randalli, we undertook preliminary genetic work (mtMuts), which confirmed Alderslade's suspicions that A. laurae (from Genbank) and our specimen $A$. randalli are identical. Therefore we consider $A$. randalli as a synonym of $A$. laurae. The species seems to be restricted to shallow, clear-water habitats (Fabricius and Alderslade 2001).

Scleraxonian corals are highly abundant in inshore areas, with only some sea fans (Melithaeidae) being more abundant offshore. Briareum stechei (Kükenthal, 1910) is highly abundant inshore and offshore and represents a new geographic record for WA (Figure 6A). Two specimens of the rare Subergorgia rubra (Thomson, 1905) were collected from Rowley Shoals, and one each from Mermaid and Imperieuse Reefs. Previously, the only Australian record was from the Coral Sea (east coast of Australia and New Caledonia) (Figure 5E). In WA, only one record exists from a CSIRO benthic biodiversity survey (Cruise SS200705), in 2007 near the Montebello Islands in the offshore Pilbara region.

Within the subordinal groups Holoxonia and Calcaxonia, the most abundant and common species were the sea whips Hicksonella princeps, Pinnigorgia flava, Rumphella aggregata within the family Gorgoniidae; Ctenocella pectinata, Dichotella gemmacea, Junceella fragilis, and J. juncea, within the family Ellisellidae, and Isis hippuris within the family Isididae (Figure 6). Only one specimen of Heliania spinescens (Gray, 1859) (Ellisellidae) was collected from Mermaid Reef, Rowley Shoals (Figure 5F). Within WA waters, this species was previously only recorded approximately $900 \mathrm{~km}$ south at Exmouth, with two specimens having been dredged from depths over $100 \mathrm{~m}$ by CSIRO (Southern Surveyor voyage SS10/2005) during benthic biodiversity surveys of the deep continental shelf and slope.

The genus Plumigorgia is reported here for the first time in WA, formerly known only from Indonesia and from the Pacific Ocean (Great Barrier Reef, the Chesterfield and Marshall Islands, Micronesia and the South China Sea) (Alderslade 1986, Fabricius and Alderslade 2001, Grasshoff and Bargibant 2001, Bryce and Poliseno 2014). The genus is represented by $P$. hydroides found at a single Hibernia Reef station occupying a small $1 \mathrm{~m}^{2}$ area (Figure $5 \mathrm{H}$ ). The only other Indian Ocean record for this species is from Indonesia. 

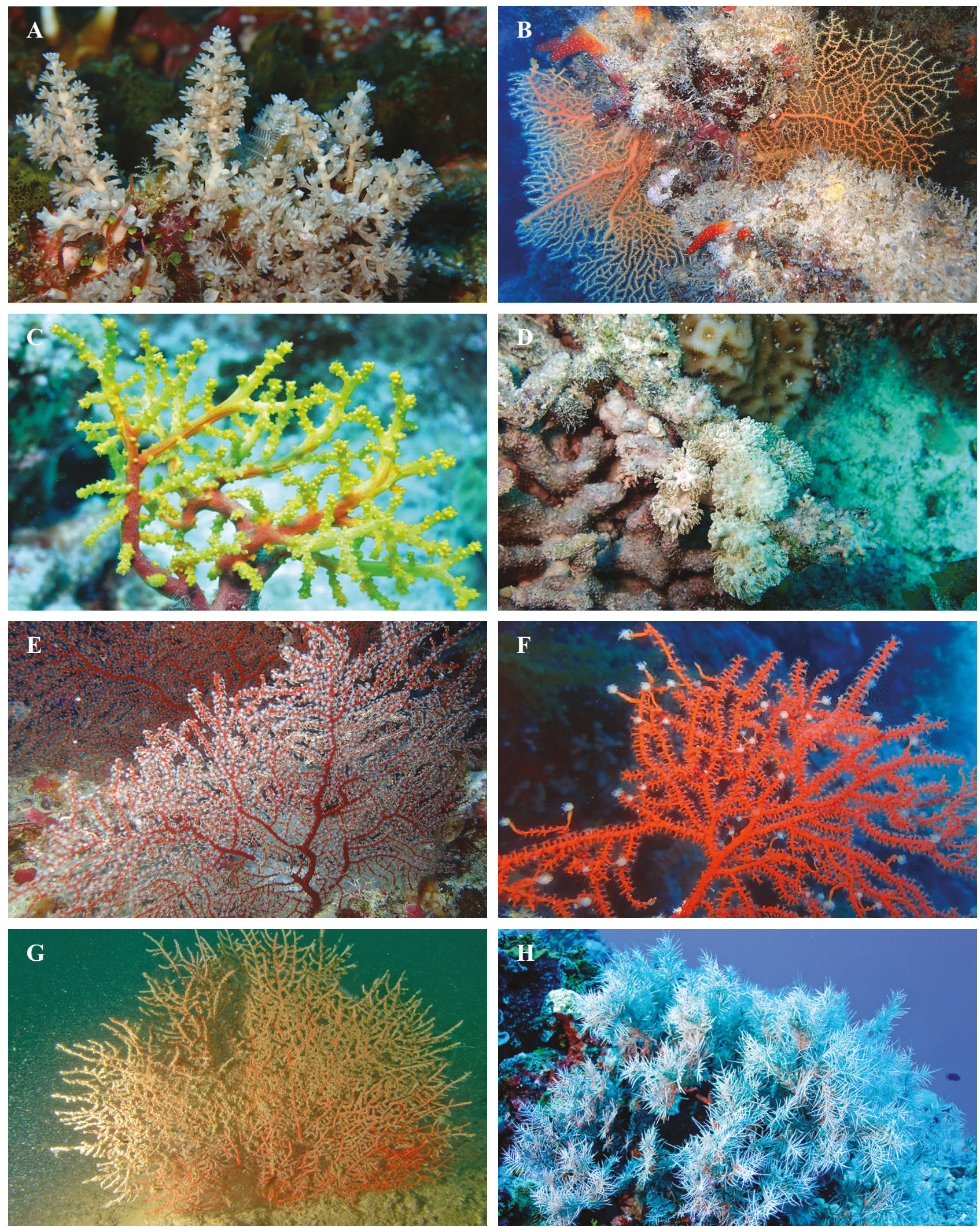

FIGURE 5 Uncommon species of Octocorallia from the Kimberley: A) Coelogorgia palmosa (Coelogorgiidae), new geographical record in Australia; B) Digitate colonies of Parasphaerasclera grayi (Parasphaerascleraeidae), new Australian record, attached to a rock together with the sea fan Annella reticulata (Subergorgiidae); C). A potentially new species of Chironephthya (Nidaliidae); D) Asterospicularia randalii (Xeniidae), new range extension for WA; E) The rare species Subergorgia rubra (Subergorgiidae); F) The rare species Heliania spinescens (Ellisellidae); G) The uncommon species Verrucella granulata (Ellisellidae); H) First record of Plumigorgia hydroides (Ifalukellidae) for Australia. 

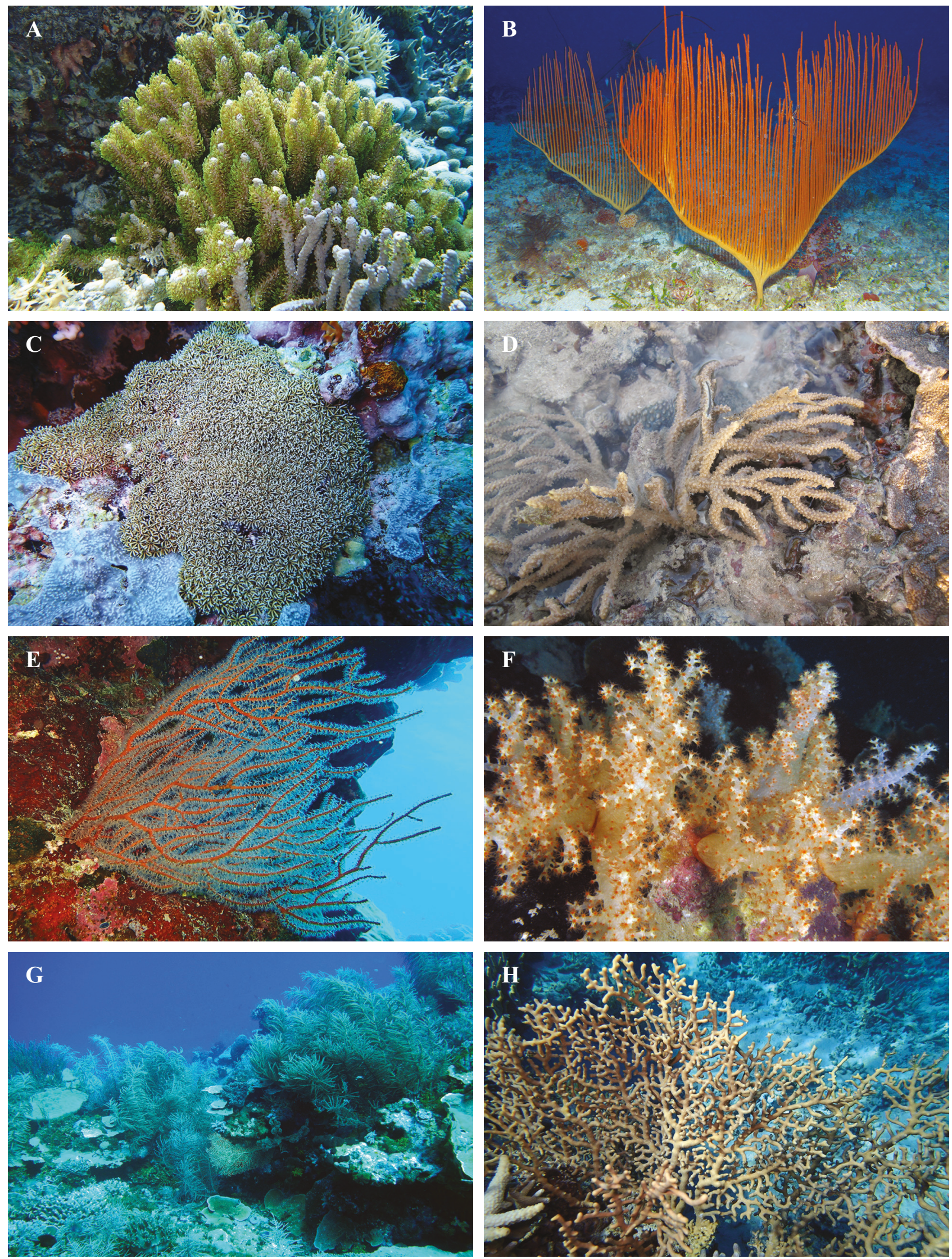

FIGURE 6

Abundant and common species of Octocorallia from the Kimberley survey area: A) Briareum stechei (Briareidae); B) Ctenocella pectinata (Ellisellidae); C) Tubipora musica (Tubiporiidae); D) Hicksonella princeps (Gorgoniidae); E) Subergorgia suberosa (Subergorgiidae); F) Scleronephthya sp. (OTU 5526); G) Pinnigorgia flava (Gorgoniidae); H) Isis hippuris (Isididae). 
TABLE 1 Species of Octocorallia (Alcyonacea and Helioporacea) recorded from the Kimberley survey area with occurrence and relative abundances. ( $x$ = present). As abundances at each transect (T1, T2) were only recorded up to 20 individuals abundances were translated into relative abundances on a rating scale of 0-5: 0 = absent; 1 = 'singletons', one or few colonies (1-3); 2 = rare (4-10); $3=$ uncommon (11-20); $4=$ common (21-100); and 5 = very common (>101) (Fabricius and Déath 2001). Morphospecies numbers are indicted within the Taxa column after the species (e.g. Carijoa sp. 5548) and are represented in both the Queensland Museum and WAM databases for future comparability.

\begin{tabular}{llll} 
Taxa & Kimberley & Ashmore and & Rowley \\
Inshore & Midshelf & $\begin{array}{l}\text { Hibernia Reefs } \\
\text { Shoals }\end{array}$ \\
\hline
\end{tabular}

\section{STOLONIFERA}

\section{Family: Clavulariidae}

Clavularia sp. 6070
Carijoa sp. 5548

Clavularia viridis (Quoy \& Gaimard, 1833)

Clavularia sp. 5363

Clavularia sp. 6067

Clavularia sp. 6068

Clavularia sp. 6069

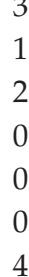

Family: Coelogorgiidae

Coelogorgia palmosa Milne-Edwards \& Haime, 1857

Family: Tubiporidae

Tubipora musica Linnaeus, 1758

2

0

0

0
0

5

\section{ALCYONIINA}

\section{Family: Alcyoniidae}

Cladiella sp. 5361

Cladiella sp. 5405

Cladiella sp. 6022

Cladiella sp. 6044

Cladiella sp. 6045

Cladiella sp. 6046

Cladiella sp. 6047

Klyxum sp. 5408

Klyxum sp. 6023

Klyxum sp. 6081

Lobophytum batarum Moser, 1919

Lobophytum crassum von Marenzeller, 1886

Lobophytum sarcophytoides Moser, 1919

Lobophytum sp. 5335

Lobophytum sp. 5506

Lobophytum sp. 5508

Lobophytum sp. 5787

Lobophytum sp. 5788

Lobophytum sp. 5789

Lobophytum sp. 6082

Lobophytum sp. 6083

Lobophytum sp. 6084

Lobophytum sp. 6085

Lobophytum sp. 6086

Lobophytum sp. 6087

Lobophytum sp. 6088

Lobophytum sp. 6089

Paraminabea aldersladei (Williams, 1992)

$$
\begin{aligned}
& 0 \\
& 0 \\
& 0 \\
& 0 \\
& 0 \\
& 4 \\
& 0
\end{aligned}
$$

2

5

0

2

4

0 


\begin{tabular}{|c|c|c|c|c|}
\hline Taxa & $\begin{array}{l}\text { Kimberley } \\
\text { Inshore }\end{array}$ & Midshelf & $\begin{array}{l}\text { Ashmore and } \\
\text { Hibernia Reefs }\end{array}$ & $\begin{array}{l}\text { Rowley } \\
\text { Shoals }\end{array}$ \\
\hline Paraminabea cf. aldersladei (6097) & 0 & 0 & 0 & 1 \\
\hline Sarcophyton glaucum (Quoy \& Gaimard, 1833) & 4 & 4 & 4 & 5 \\
\hline Sarcophyton trocheliophorum von Marenzeller, 1886 & 0 & 1 & 0 & 0 \\
\hline Sarcophyton sp. 5395 & 4 & 0 & 0 & 0 \\
\hline Sarcophyton sp. 5396 & 2 & 0 & 0 & 0 \\
\hline Sarcophyton sp. 5397 & 1 & 0 & 0 & 0 \\
\hline Sarcophyton sp. 5398 & 1 & 0 & 0 & 0 \\
\hline Sarcophyton sp. 5399 & 1 & 0 & 0 & 0 \\
\hline Sarcophyton sp. 5400 & 1 & 0 & 0 & 0 \\
\hline Sarcophyton sp. 5409 & 2 & 0 & 0 & 0 \\
\hline Sarcophyton sp. 5412 & 1 & 0 & 0 & 0 \\
\hline Sarcophyton sp. 5786 & 4 & 0 & 0 & 0 \\
\hline Sarcophyton sp. 6102 & 0 & 0 & 0 & 2 \\
\hline Sarcophyton sp. 6103 & 0 & 0 & 0 & 1 \\
\hline Sarcophyton sp. 6104 & 0 & 0 & 0 & 2 \\
\hline Sarcophyton sp. 6105 & 0 & 0 & 0 & 1 \\
\hline Sarcophyton sp. 6106 & 0 & 0 & 1 & 0 \\
\hline Sarcophyton sp. 6107 & 0 & 0 & 4 & 0 \\
\hline Sarcophyton sp. 6108 & 0 & 0 & 4 & 0 \\
\hline Sarcophyton sp. 6109 & 0 & 0 & 4 & 0 \\
\hline Sarcophyton sp. 6110 & 0 & 1 & 4 & 0 \\
\hline Sarcophyton sp. 6111 & 0 & 0 & 4 & 0 \\
\hline Sarcophyton sp. 6050 & 1 & 1 & 0 & 0 \\
\hline Sarcophyton sp. 6051 & 1 & 0 & 0 & 0 \\
\hline Sinularia brassica May, 1898 & 5 & 0 & 4 & 3 \\
\hline Sinularia (Dampia) pocilloporaeformis Alderslade, 1983 & 2 & 0 & 1 & 1 \\
\hline Sinularia spp. & 5 & 3 & 5 & 5 \\
\hline \multicolumn{5}{|l|}{ Family: Nephtheidae } \\
\hline Capnella garetti Verseveldt, 1977 & 4 & 2 & 4 & 0 \\
\hline Capnella imbricata (Quoy \& Gaimard, 1833) & 1 & 0 & 5 & 0 \\
\hline Capnella sp. 5472 & 2 & 0 & 0 & 0 \\
\hline Capnella sp. 6061 & 0 & 0 & 2 & 0 \\
\hline Chromonephthea spp. & 4 & 0 & 0 & 0 \\
\hline Dendronephthya spp. & 5 & 2 & 4 & 5 \\
\hline Lemnalia sp. 5392 & 1 & 1 & 0 & 0 \\
\hline Lemnalia sp. 6125 & 0 & 0 & 4 & 0 \\
\hline Lemnalia sp. 6126 & 0 & 0 & 5 & 0 \\
\hline Lemnalia sp. 6127 & 0 & 1 & 4 & 3 \\
\hline Lemnalia sp. 6128 & 0 & 0 & 1 & 0 \\
\hline Nephthea sp. 6129 & 0 & 3 & 4 & 0 \\
\hline Nephthea sp. 5393 & 5 & 2 & 5 & 1 \\
\hline Nephthea sp. 5522 & 1 & 4 & 0 & 0 \\
\hline Nephthea sp. 5523 & 1 & 0 & 0 & 0 \\
\hline Nephthea sp. 6092 & 0 & 0 & 0 & 5 \\
\hline Nephthea sp. 6093 & 0 & 0 & 0 & 1 \\
\hline Nephthea sp. 6094 & 0 & 0 & 0 & 2 \\
\hline Paralemnalia sp. 5401 & 4 & 0 & 5 & 4 \\
\hline Paralemnalia sp. 5766 & 1 & 0 & 0 & 0 \\
\hline Scleronephthya sp. 5526 & 5 & 4 & 2 & 1 \\
\hline
\end{tabular}




\begin{tabular}{|c|c|c|c|c|}
\hline Taxa & $\begin{array}{l}\text { Kimberley } \\
\text { Inshore }\end{array}$ & Midshelf & $\begin{array}{l}\text { Ashmore and } \\
\text { Hibernia Reefs }\end{array}$ & $\begin{array}{l}\text { Rowley } \\
\text { Shoals }\end{array}$ \\
\hline Scleronephthya sp. 6112 & 0 & 0 & 0 & 2 \\
\hline Scleronephthya sp. 6113 & 0 & 0 & 0 & 1 \\
\hline Scleronephthya sp. 6114 & 1 & 0 & 0 & 0 \\
\hline Stereonephthya sp. 5438 & 1 & 0 & 0 & 0 \\
\hline Stereonephthya sp. 5767 & 4 & 0 & 0 & 0 \\
\hline Stereonephthya sp. 6035 & 0 & 1 & 2 & 0 \\
\hline Stereonephthya sp. 6036 & 0 & 0 & 4 & 0 \\
\hline Stereonephthya sp. 6037 & 0 & 1 & 4 & 0 \\
\hline Stereonephthya sp. 6038 & 0 & 0 & 4 & 0 \\
\hline Stereonephthya sp. 6121 & 0 & 0 & 0 & 4 \\
\hline Stereonephthya sp. 6122 & 0 & 0 & 0 & 4 \\
\hline Stereonephthya sp. 6123 & 0 & 4 & 0 & 0 \\
\hline \multicolumn{5}{|l|}{ Family: Parasphaerascleridae } \\
\hline Parasphaerasclera grayi (Thomson \& Dean, 1931) & 2 & 0 & 0 & 2 \\
\hline Parasphaerasclera kimberleyensis Bryce et. al., 2015 & 1 & 0 & 0 & 0 \\
\hline
\end{tabular}

\section{Family: Nidaliidae}

Chironephthya sp. 6032

Chironephthya sp. 6033

Chironephthya sp. 6062

Chironephthya sp. 6063

Chironephthya sp. 6064

Chironephthya sp. 6065

Chironephthya sp. 6066

Nephthyigorgia kükenthali Broch, 1916

Siphonogorgia sp. 6034

Siphonogorgia sp. 6115

Siphonogorgia sp. 6116

Siphonogorgia sp. 6117

Siphonogorgia sp. 6118

Siphonogorgia sp. 6119

Siphonogorgia sp. 6120

$\begin{array}{ll}0 & 0 \\ 0 & 0 \\ 0 & 0 \\ 0 & 0 \\ 0 & 0 \\ 0 & 0 \\ 1 & 0 \\ 1 & 0 \\ 0 & 0 \\ 0 & 0 \\ 0 & 0 \\ 0 & 0 \\ 0 & 0 \\ 0 & 0 \\ 0 & 0\end{array}$

\section{Family: Xeniidae}

Anthelia sp. 5490

Anthelia sp. 6054

Asterospicularia randalli Gawel, 1976

Heteroxenia sp. 5769

Heteroxenia sp. 6080

Sansibia sp. 5365

Sansibia sp. 5367

Sansibia sp. 6100

Sansibia sp. 6101

Sympodium sp. 6124

Xenia spp.

1
0
0
1
1
1
4
0
1
0
5

\section{SCLERAXONIA}

\section{Family: Briareidae}

Briareum stechei (Kükenthal, 1908)

Briareum violaceum (Quoy \& Gaimard, 1833) 


\begin{tabular}{|c|c|c|c|}
\hline Taxa & $\begin{array}{l}\text { Kimberley } \\
\text { Inshore }\end{array}$ & Midshelf & $\begin{array}{l}\text { Ashmore and } \\
\text { Hibernia Reefs }\end{array}$ \\
\hline \multicolumn{4}{|l|}{ Family: Anthothelidae } \\
\hline Alertigorgia mjöbergi Broch, 1916 & 4 & 0 & 0 \\
\hline Iciligorgia brunnea (Nutting, 1911) & 3 & 0 & 1 \\
\hline \multicolumn{4}{|l|}{ Family: Subergorgiidae } \\
\hline Annella reticulata (Ellis \& Solander, 1786) & 3 & 0 & 1 \\
\hline Subergorgia rubra (Thomson, 1905) & 0 & 0 & 0 \\
\hline Subergorgia suberosa (Pallas, 1766) & 5 & 1 & 4 \\
\hline \multicolumn{4}{|l|}{ Family: Melithaeidae } \\
\hline Melithaea sp. 5337 & 1 & 1 & 0 \\
\hline Melithaea sp. 5338 & 0 & 0 & 4 \\
\hline Melithaea sp. 5339 & 1 & 1 & 4 \\
\hline Melithaea sp. 5362 & 2 & 0 & 0 \\
\hline Melithaea sp. 5425 & 4 & 1 & 4 \\
\hline Melithaea sp. 5426 & 3 & 1 & 4 \\
\hline Melithaea sp. 5427 & 4 & 0 & 4 \\
\hline Melithaea sp. 5470 & 1 & 0 & 0 \\
\hline Melithaea sp. 5410 & 1 & 0 & 0 \\
\hline Melithaea sp. 5511 & 1 & 0 & 0 \\
\hline Melithaea sp. 5600 & 4 & 4 & 0 \\
\hline Melithaea sp. 5773 & 1 & 0 & 0 \\
\hline Melithaea sp. 5774 & 2 & 0 & 0 \\
\hline Melithaea sp. 5475 & 2 & 0 & 0 \\
\hline Melithaea sp. 5776 & 4 & 0 & 0 \\
\hline Melithaea sp. 5777 & 2 & 0 & 0 \\
\hline Melithaea sp. 6039 & 0 & 0 & 3 \\
\hline Melithaea sp. 6040 & 0 & 1 & 4 \\
\hline Melithaea sp. 6041 & 0 & 0 & 4 \\
\hline Melithaea sp. 6043 & 0 & 1 & 0 \\
\hline
\end{tabular}

\section{HOLOXONIA}

Family: Acanthogorgiidae

Acanthogorgia sp. 5403

Acanthogorgia sp. 6025

Acanthogorgia sp. 6052

Acanthogorgia sp. 6053

Anthogorgia sp. 5488

Family: Plexauridae

Astrogorgia sp. 5487

Astrogorgia sp. 6026

Astrogorgia sp. 6031

Astrogorgia sp. 6055

Astrogorgia sp. 6056

Astrogorgia sp. 6057

Astrogorgia sp. 6058

Astrogorgia sp. 6059

Bebryce sp. 5491

Bebryce sp. 6024

Echinogorgia sp. 5495

Echinogorgia sp. 5772

Echinogorgia sp. 6071

Kimberley

wley

Shoals 


\begin{tabular}{|c|c|c|c|c|}
\hline Taxa & $\begin{array}{l}\text { Kimberley } \\
\text { Inshore }\end{array}$ & Midshelf & $\begin{array}{l}\text { Ashmore and } \\
\text { Hibernia Reefs }\end{array}$ & $\begin{array}{l}\text { Rowley } \\
\text { Shoals }\end{array}$ \\
\hline Echinogorgia sp. 6072 & 1 & 0 & 0 & 0 \\
\hline Echinogorgia sp. 6073 & 1 & 0 & 0 & 0 \\
\hline Echinomuricea sp. 6074 & 1 & 0 & 0 & 0 \\
\hline Euplexaura sp. 5441 & 3 & 0 & 1 & 0 \\
\hline Euplexaura sp. 5500 & 4 & 0 & 0 & 0 \\
\hline Euplexaura sp. 6078 & 0 & 0 & 0 & 1 \\
\hline Menella sp. 5507 & 4 & 0 & 0 & 0 \\
\hline Menella sp. 5771 & 2 & 0 & 0 & 0 \\
\hline Menella sp. 6090 & 0 & 0 & 0 & 1 \\
\hline Menella sp. 6091 & 1 & 0 & 0 & 0 \\
\hline Paracis sp. 6095 & 0 & 0 & 0 & 1 \\
\hline Paracis sp. 6096 & 0 & 0 & 0 & 1 \\
\hline Paraplexaura sp. 5494 & 2 & 2 & 0 & 0 \\
\hline Paraplexaura sp. 5496 & 1 & 0 & 0 & 0 \\
\hline Paraplexaura sp. 5497 & 1 & 0 & 0 & 0 \\
\hline Paraplexaura sp. 5498 & 1 & 0 & 0 & 0 \\
\hline Paraplexaura sp. 6030 & 0 & 0 & 4 & 0 \\
\hline Paraplexaura sp. 6098 & 0 & 0 & 0 & 2 \\
\hline Paraplexaura sp. 6099 & 1 & 0 & 0 & 0 \\
\hline Villogorgia sp. 6027 & 0 & 0 & 4 & 1 \\
\hline \multicolumn{5}{|l|}{ Family: Gorgoniidae } \\
\hline Hicksonella princeps Nutting, 1910 & 2 & 2 & 5 & 1 \\
\hline Pinnigorgia flava (Nutting, 1910) & 0 & 0 & 4 & 0 \\
\hline Pseudopterogorgia australiensis (Ridley, 1884) & 3 & 0 & 0 & 0 \\
\hline Rumphella aggregata (Nutting, 1910) & 5 & 2 & 1 & 3 \\
\hline
\end{tabular}

\section{CALCAXONIA}

\section{Family: Ellisellidae}

Ctenocella pectinata (Pallas, 1766) 4

Dichotella gemmacea (Milne Edwards \& Haime, 1857)

Dichotella sp. 5779

Dichotella sp. 5780

Dichotella sp. 5781

Ellisella ceratophyta (Linnaeus, 1758)

Ellisella sp. 6028

Ellisella sp. 6076

Ellisella sp. 6077

Heliania spinescens (Gray, 1859)

Junceella fragilis (Ridley, 1884)

Junceella juncea (Pallas, 1766)

Verrucella granulata (Esper, 1788)

Viminella sp. 6029

Family: Ifalukellidae

Plumigorgia hydroides Nutting, 1910

Family: Isididae

Isis hippuris Linnaeus, 1758

\section{HELIOPORACEA}

\section{Family: Helioporidae}




\section{DESCRIPTION OF SOFT CORAL ALPHA BIODIVERSITY AND MAJOR SPATIAL PATTERNS}

Alpha diversity was investigated for the 177 octocoral stations surveyed (Figure 7) and separately for the 153 survey stations where transects were conducted (Figure 8). The former included species information beyond the transects and stations where biodiversity only collections were conducted to $30 \mathrm{~m}$ depth (Figure 7). The latter represented species richness along a $100 \mathrm{~m}$ transect set at approximately $12 \mathrm{~m}$ depth (Figure 8). Alpha diversity analyses demonstrated strong inshore/offshore patterns and a latitudinal zonation along the inshore Kimberley. Soft coral species richness varied between stations and localities, but was clearly higher at deeper stations and at northern offshore stations when compared to inshore locations (Table 1). Species richness across all stations ranged from $0-31$ species per survey station, and 0-28 species per transect (Figures 7 and 8 respectively). Intertidal stations were consistently depauperate, while reef front stations had relatively high species richness. The highest alpha diversity $(>23)$ in the survey area was found offshore at Ashmore and Hibernia Reefs and inshore at Jamieson and Heritage Reefs and Patricia Island (Figure 7). High species richness (16-22) was recorded from Ashmore and Hibernia Reefs (offshore north), from the deeper stations at the Rowley Shoals (offshore south), from Heywood Shoals (midshelf), Cassini, Maret, Montalivet and Condillac Islands and Jamieson Reef (inshore north), and from De Freycinet and White Islands and Black Rock (middle inshore) (Figure 7). Offshore, the highest transect species richness with 31 species was found on a steep fore reef slope on the north side of Ashmore Reef (station 130K/13). Generally, biodiversity increased with decreasing longitude and latitude. The offshore biodiversity 'hotspots' of Ashmore and Hibernia Reefs were linked to the coastal Bonaparte Archipelago 'hotspots' via the diverse midshelf region. Inshore, transect species richness peaked at the northern extremity of the Bonaparte Archipelago with 27 species from a fore reef slope at Jamieson Reef (station 110/K12).

A comparison of soft coral diversity data between all transects and stations (to a depth of $30 \mathrm{~m}$ ) provided a more complete picture of trends and 'hotspots', than transect data alone. This was due to the inclusion of 'biodiversity stations' and sampling at greater depths (Figures 7 and 8). The biodiversity stations highlighted the importance of the midshelf habitats as biotic links between offshore and inshore habitats. The importance of collecting at greater depths, which was especially apparent at the Rowley Shoals, cannot be understated (Figure 8). Overall biodiversity was always higher than 'transect biodiversity', with some stations having a distinct low species richness or no soft corals at all on the transects, while overall richness could be relatively high. For example, at Robroy Reef (118/K12) only three species and at Brue Reef (85/K11) only two species were recorded on the transects, yet station species richness was 15 and 13 respectively.

\section{DESCRIPTION OF MAJOR PATTERNS OF SOFT CORAL COMMUNITY COMPOSITION, INCLUDING SPATIAL PATTERNS}

A matrix dendrogram was used to aggregate the 153 transects into groups based on similarity of octocoral composition and resulted in five clusters (C1-C5; Figure 9).

The octocoral assemblages were spatially associated and displayed a clear pattern of benthic community structure within the survey area. To further visualise and define these spatial patterns the clusters were depicted on the Project Area map (Figure 10). The five clusters (C1-C5) were strongly related to environmental conditions and characterised by spatial patterns of habitat occurrences (Table 2). Cluster 1 (green) consists of $83 \%$ of inshore stations from Montgomery Reef, Adele Island and Champagney Islands in the southern end of the inshore area. This group consisted of $61 \%$ intertidal sites and was dominated by the soft coral genera Sarcophyton and Clavularia. Cluster 2 (purple) is represented by $81 \%$ of inshore subtidal stations along the entire length of the inshore, as well as four midshelf subtidal stations from Browse Island and two offshore subtidal lagoonal stations. It was comprised of a suite of octocoral species, with indicator species including a diverse assemblage of sea whips (Junceella fragilis, Junceella juncea, Dichotella gemmacea, Ctenocella pectinata, Pseudopterogorgia australiensis, Rumphella aggregata, Subergorgia suberosa) and soft coral families, such as Nephtheidae (Dendronephthya, Chromonephthea, Scleronephthya), Plexauridae (Euplexaura, Menella, Astrogorgia) and Briareidae. Cluster 3 (brown) includes offshore Rowley Shoals sites as well as two inshore sites (Fraser Island and Woodward Island). Cluster 4 (blue) is composed of $95 \%$ of the inshore intertidal stations, with a high clustering of stations at the northern inshore. Interestingly, there appears to be a soft coral connection to the offshore area with the remaining $5 \%$ of stations 


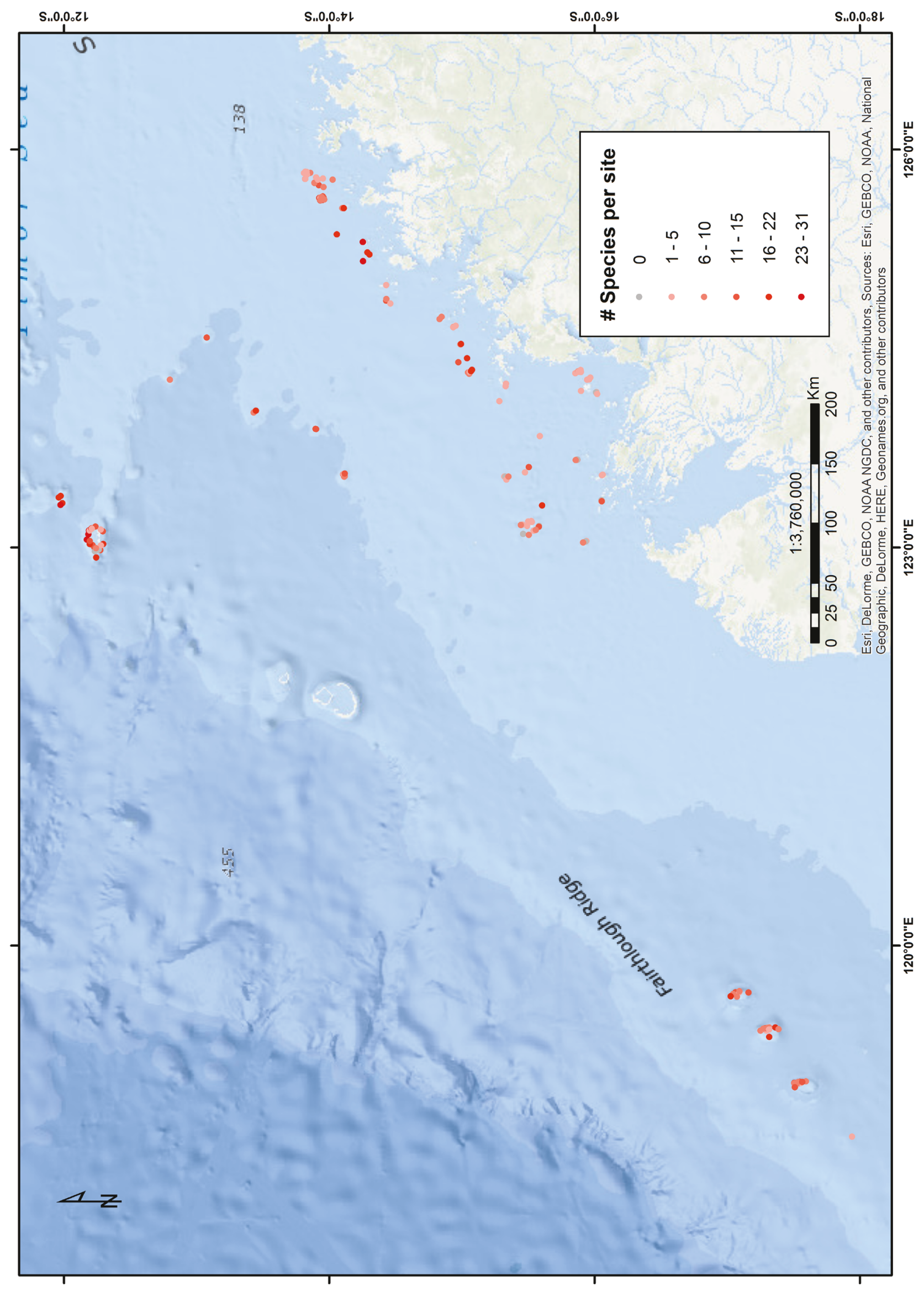

FIGURE 7 Alpha-diversity of all octocoral sampling stations. 
$5.0,0 . Z$

S..0.0.tr

S..0.0.9l

$5.0 .0 \circ 81$

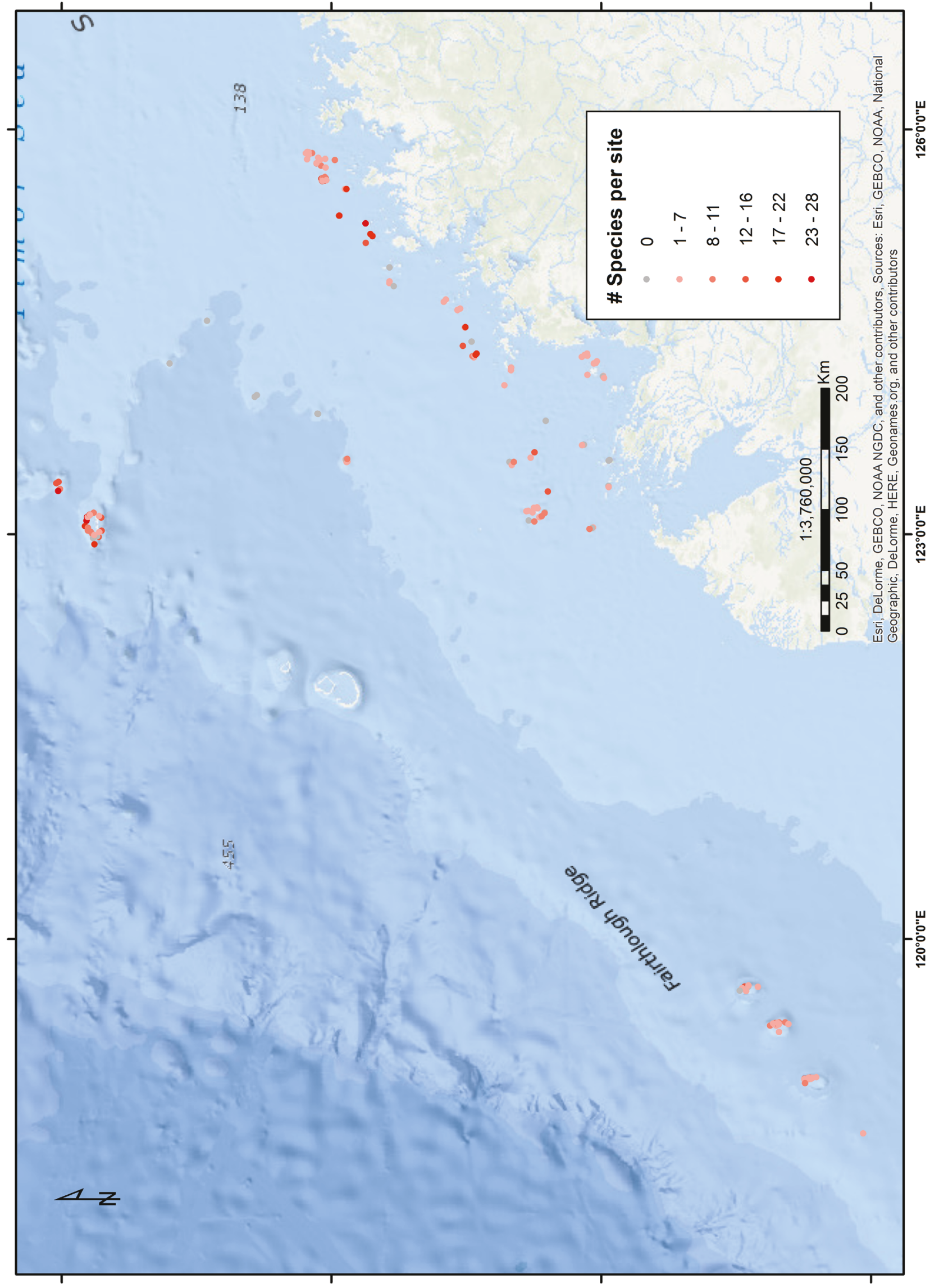


being composed of a midshelf station (Browse Island), four intertidal and one lagoonal station at Ashmore Reef in the far north of the survey area. This group was dominated by the soft coral genera Tubipora, Lobophytum and Capnella. Offshore, Ashmore and Hibernia Reefs appeared distinct (Cluster 5 - pink) and were distinguished from the other groups by a complex and diverse suite of soft corals, sea whips and sea fans.

\section{DISCUSSION}

\section{CORAL SPECIES AND ASSEMBLAGES}

The large Project Area surveyed in this study, approximately $476,000 \mathrm{~km}^{2}$, provided considerable latitudinal and longitudinal gradients, and spanned shallow inshore waters $(<30 \mathrm{~m})$ across a wide continental shelf to the continental edge $(>200 \mathrm{~m}$ depth). The complex environmental differences and drivers occurring in the area, such as the origin of seabed sediments, nutrient levels and turbidity, tidal regimes and currents, as well as differences in levels of connectivity, account for distinctive offshore and inshore reefal communities, and this was clearly reflected in octocoral species community composition and distribution (Wilson 2014; Bryce et al. 2018). Main vectors driving the cross shelf distribution of benthic organisms in the Project Area were the high cover of rock, silt and hard corals at the inshore locations, and the high cover of turf algae, coralline algae, sand, rubble, and soft corals in the offshore regions. Habitat separation of benthic organisms in the intertidal was driven by rock and silt, turf algae and macro-algae in the intertidal areas and high cover of coralline algae, hard and soft corals in the subtidal areas (Richards et al. 2018). When sites were compared using proportional composition of octocoral taxa, clear associations between octocoral assemblages and habitats were apparent. Associations between octocoral species communities and localities were apparent with strong inshore versus offshore patterns and a latitudinal zonation along the Kimberley inshore.

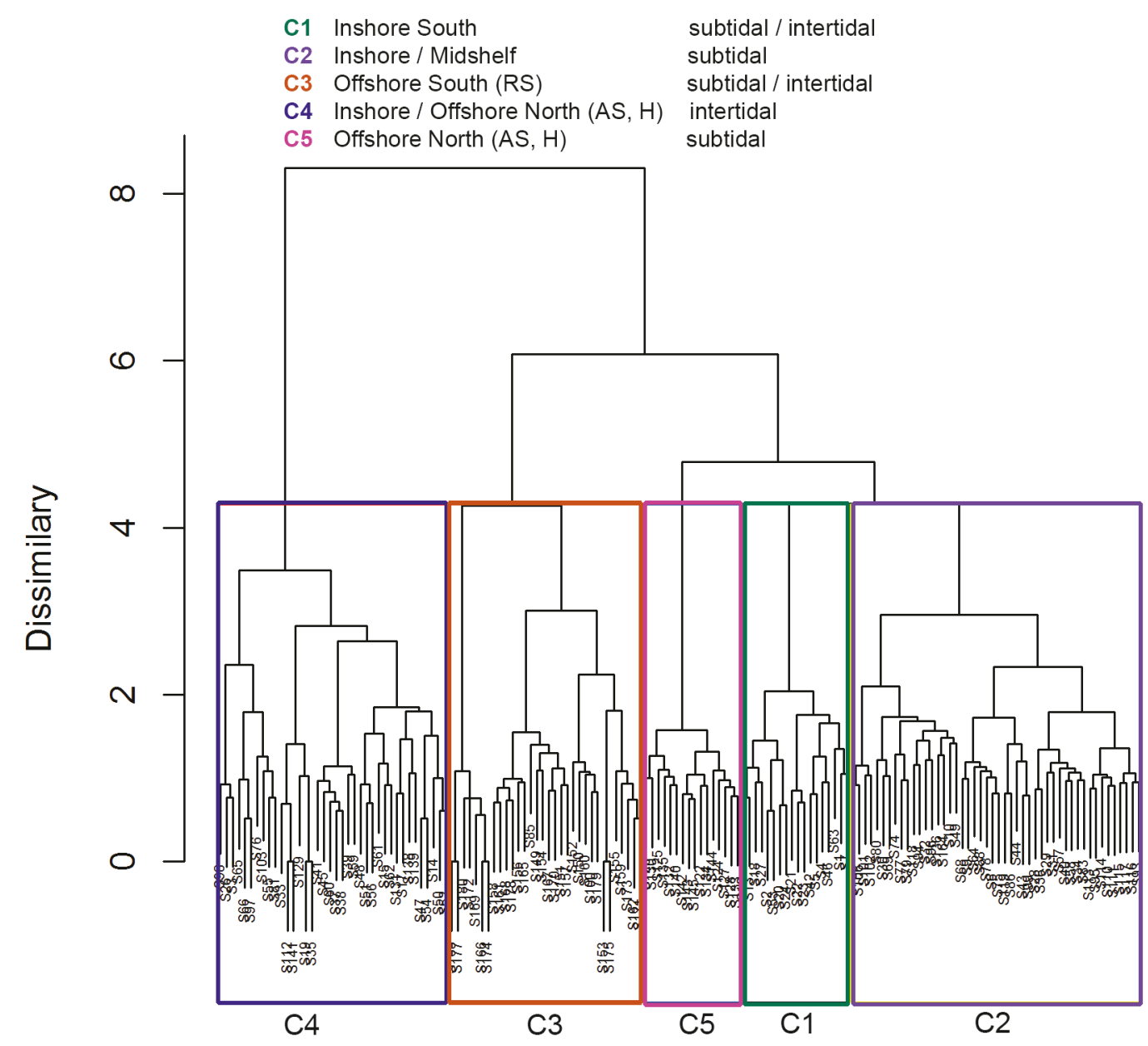

FIGURE 9 Dendrogram depicting transects grouped according to their similarity in octocoral species composition. Dendrogram based on a hierarchical cluster analysis resulted in 5 clusters (C1-C5). RS = Rowley Shoals, $\mathrm{AS}=$ Ashmore Reef and $\mathrm{H}=$ Hibernia Reef. 


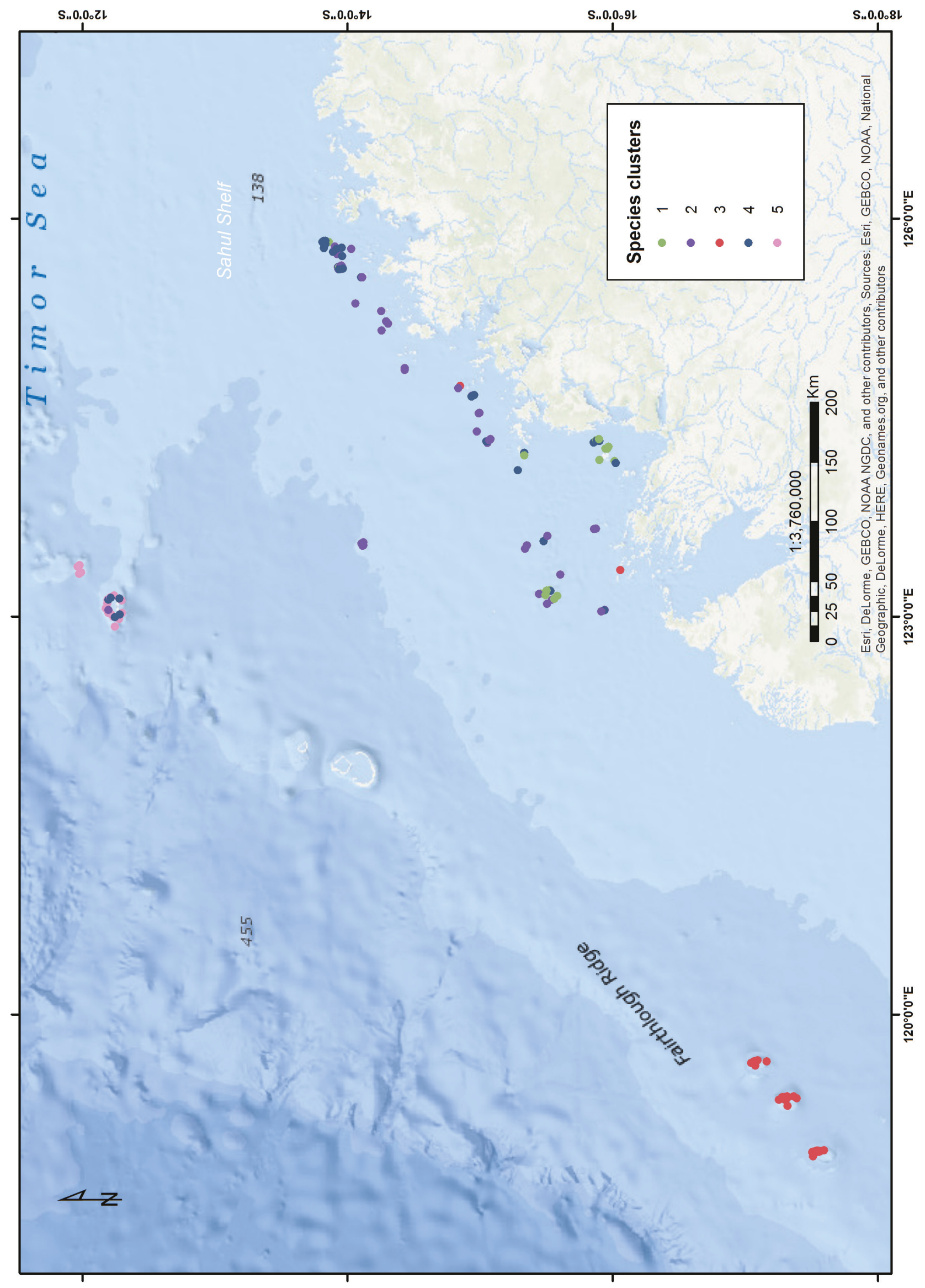

FIGURE 10 Cluster species composition for each octocoral transect. The five clusters from the dendrogram C1-C5 in Figure 9 were plotted on the map to visualise spatial patterns. 
Octocoral species assemblages can be grouped into 5 regions: 1) Inshore stations in the southern end of the survey area (C1); 2) inshore subtidal stations along the central inshore section including the midshelf subtidal sites (C2); 3) inshore and offshore intertidal stations within the northern end of the survey area (C4); the Rowley Shoals (C3) and 5) Ashmore and Hibernia Reefs (C5) (see Figure 10). Species patterns revealed a clear connection between the northern inshore and offshore areas, as well as strong associations between the middle inshore area and the midshelf stations. Surprisingly, lagoonal sites within atolls did not present as a particular habitat type. The three atolls of the Rowley Shoals in the offshore south exhibited a homogeneous and distinct community, which is in agreement with a previous octocoral biodiversity survey (Fabricius 2008).

Octocoral richness also varied across stations. Thirty-nine percent of octocoral species in the survey area were found over the entire inshore and offshore range of habitats, while the remaining species were either restricted to turbid inshore reefs or the clear waters of the shelf edge atolls. Nearly half of all octocoral species (45\%) were exclusively present at clear offshore and midshelf sites, while only a few species $(16 \%)$ were restricted to turbid inshore areas. Nevertheless, the latter were all singletons and may be globally rare and highly restricted in their distribution, but further collecting is required. This pattern could be attributed to the direct pelagic connectivity of these shelf edge reefs with the species rich bioregions of eastern Indonesia, while inshore, reef connectivity is controlled by local currents driven by seasonal wind and tidal flow (Wilson 2014).

Different genera of octocorals demonstrate different habitat preferences, with growth strongly contrasting between locations. Intertidal stations were less diverse than subtidal stations and were characterised to a large extent by the genera Tubipora, Clavularia, Sarcophyton, Lobophytum, Sinularia, Capnella, and Xenia. For soft corals, intertidal environments are stressful, subject to strong wave and tidal action and desiccation during long exposure times at low tides. Soft coral body plans for these harsh sites were predominantly encrusting, short lobed or massive, to flat species. Inshore subtidal stations were comprised of a diverse assemblage of sea whips (Junceella fragilis, Junceella juncea, Dichotella gemmacea, Ctenocella pectinata, Pseudopterogorgia australiensis, Rumphella aggregata, Subergorgia suberosa) and soft coral families (Alcyoniidae, Nephtheidae, Plexauridae and Briareidae). Northern offshore localities were more species rich than the south and this can be attributed to the flow of tropical water from the Western Pacific arriving at suitable habitats during recruitment (Wilson 2014). Relative abundances were similar offshore and inshore, with $40 \%$ of the octocorals represented by one or a few colonies, $14-17 \%$ rare, $10-13 \%$ uncommon, $22-25 \%$ common and $8-11 \%$ very common (Table 1 ).

All major groups and genera of Indo-Pacific octocorals were represented (Fabricius and Alderslade 2001). This comprehensive survey revealed many new geographic records, range extensions and new, and potentially new, species. This can be attributed to the large survey area with a diverse array of habitats incorporating less accessible areas, such as reef overhangs and exposed sites, which may not have been sampled in the past. A similar trend has been observed in sampling programs of other areas poorly sampled for octocorals, such as the Pilbara and King George River estuary in north-western Australia (Keesing et al. 2004, Pitcher et. al. 2016).

Offshore biodiversity 'hotspots', such as Ashmore and Hibernia Reefs, were linked to the inshore Bonaparte Archipelago 'hotspots'(Robroy Reefs, Jamieson and Heritage Reef, Maret, and Montalivet Islands and Condillac and Patricia Islands) via the diverse midshelf region (Browse Island and the several visited shoals).

Alcyoniidae is a dominant family in the Kimberley region consistent with patterns of global and regional Australian soft coral diversity. The family has been reported from other IndoPacific locations, such as Palau eastward to the Great Barrier Reef and the east coast of Africa (Benayahu 1985; Fabricius and Déath 2001, Benayahu et al. 2003, Fabricius et al. 2007, Chanmethakul et al. 2010, Benayahu et al. 2012). Alcyoniidae are widely distributed in all habitat types from the intertidal to greater depths, and can cover large areas. They are able to survive harsh conditions, such as heavy sediment loads and extended exposure, which is an important advantage for surviving extreme Kimberley conditions.

Dominant families were Nephtheidae, Plexauridae, and Melithaeidae, and speciose families included Nidaliidae and Ellisellidae (Figure 3B). Melithaeidae, Plexauridae, Nidaliidae, Ellisellidae, and several genera among the family Nephtheidae are heterotrophic suspension feeders that depend on currents to transport food particles towards their polyps. All other families in this study are represented by only a few species and 
are generally species limited taxa, but they do incorporate new species and range extensions, and are therefore important contributors to the overall knowledge of regional biodiversity.

Marine filter feeding communities are dominated by soft corals, sea fans, sponges and ascidians and can form extensive garden-like habitats characterised by high biomass and diversity. These benthic organisms are vital components of nearshore and offshore tropical ecosystems through their contribution to carbon cycling and productivity, provision of habitat for an array of associated biodiversity, and play a vital role as nursery areas. Octocoral transect cover peaked at $30 \%$ at only a few stations. Generally, octocoral cover was low, attributable to an artefact of sampling where the majority of transects were placed on coral reef sites and at a depth comparable with other coral studies. An example is a mid-littoral reef platform at Long Reef (station $56 /$ K10) with zero octocoral cover on the transect, but the reef platform bordering the transect had the octocoral Tubipora as the dominant benthic organism with 28\% cover (Richards et al. 2013). Benthic studies of tropical reefs are dominated by hard corals, and a focus on coral cover is largely defined by scleractinian cover (Przeslawski et al. 2008; Edmunds et al. 2014). However, a more balanced approach needs to also include the examination of other benthic taxa including octocorals, which not only play a dominant role in extant benthic community structure, but may well populate and dominate future reefs (Blakeway and Radford 2004; Hoegh-Guldberg et al. 2007; Hughes et al. 2010; Edmunds et al. 2014; Baum et al. 2016). Numerous studies over the last several decades have documented declines in the abundance of reef building corals after disturbance events and a phase shift to dominance by fleshy, non-calcifying invertebrates (Benayahu and Loya 1981; Bradbury and Mundy 1989; Fabricius 1996, 1997, 1998; Fox et al. 2003; Przeslawski 2008; Ruzicka et al. 2013; Lenz et al. 2015; Smith et al. 2016). The faunistic importance of octocorals as key contributors to ecosystem services in the Kimberley illustrates the need for further soft coral surveys that will encompass the full spectrum of reef types and habitats, especially inter-reefal and understudied environments, such as soft sediment and sandy areas.

\section{MANAGEMENT IMPLICATIONS}

To date, this study is the most comprehensive study for soft corals undertaken within the Kimberley. This synthesis of soft coral data will facilitate future surveys in the region and will help fill remaining knowledge gaps. Comprehensive surveys in remote areas, such as the Kimberley marine environments, are important to provide a clearer understanding of soft coral taxonomy, systematics and distributional patterns in tropical Indo-Pacific marine environments. These data provide valuable insight into the regions biodiversity and habitats, with important implications for marine area protection, but future consideration should be given to environmental parameters, sampling effort and methodology, and geomorphic zones in order to gain a more comprehensive understanding (Fabricius 2008; Keesing et al. 2011; Pitcher et al. 2016; Fromont et al. 2016). This work underpins the hypothesis that the west coast of Australia is a unique ecosystem supporting a rich and diverse octocoral fauna (Marsh 1993; Bryce 2009; Masini et al. 2009; Wilson 2013; Richards et al. 2013), and further investigations will enhance our understanding of these environments. Meaningful ecological management areas, for potential estimates of habitat loss and to assess long term changes in community structures, can be defined by providing better ecological information and by locating appropriate reference sites within similar community types (EPA 2004; Pitcher et al. 2016; Moore et al. 2016). Long term monitoring of environmental conditions and impacting pressures is also essential to evaluate management effectiveness and to inform future research and decision making (Department of Wildlife 2016). These survey results on octocoral assemblages within the Kimberley will provide useful data for future monitoring and environmental management of marine areas, in particular where impact monitoring is proposed.

\section{ACKNOWLEDGEMENTS}

The Western Australian Museum and its partner agencies respectfully acknowledge the Traditional Custodians of the Kimberley land and sea country, of Elders past and present, and in particular the Dambimangari and Wunambal Gaambera peoples, for collections made on their sea country.

Sincere thanks to various agencies that supported fieldwork, including Woodside Energy Ltd. through the Western Australian Museum / Woodside Collection Project (Kimberley) and the Queensland Museum under the National Taxonomic Research Grant (ABRS 209-05). Thanks to our taxonomic colleague Phil Alderslade for his help with species identification and his invaluable comments on this manuscript. The crew of the Kimberley Quest, Merrick Ekins, and Monique Grol are thanked for field assistance and Kate Dawson for laboratory assistance. Angelo Poliseno, University of Munich, is thanked for genetic work. The comments of two anonymous reviewers improved this manuscript. 
TABLE 2 Survey stations per cluster ordered to match Figures 9 and 10 in this paper and Figure 2 in Bryce et al. 2018.

\section{C1: Offshore intertidal walking}

$1 \quad$ Adele Island

2 Adele Island

$4 \quad$ Adele Island

$5 \quad$ Adele Island

$7 \quad$ Adele Island

12 Adele Island

13 Adele Island

18 Montgomery Reef

20 Montgomery Reef

21 Montgomery Reef

22 Montgomery Reef

23 Montgomery Reef

24 Montgomery Reef

27 Montgomery Reef

42 Cassini Island

$46 \quad$ Long Reef

53 Long Reef

63 Champagney Islands

\section{C2: Midshelf intertidal walking}

6 Adele Island

$9 \quad$ Adele Island

10 Adele Island

Cassini Island

Cassini Island

Cassini Island

Cassini Island

Cassini Island
Inshore subtidal

Inshore subtidal

Inshore subtidal

Inshore subtidal

Inshore intertidal walking

Inshore subtidal

Inshore subtidal

Inshore intertidal walking

Inshore intertidal walking

Inshore intertidal walking

Inshore intertidal walking

Inshore intertidal walking

Inshore intertidal walking

Inshore intertidal walking

Inshore intertidal dive/snorkel

Inshore intertidal dive/snorkel

Inshore subtidal

Inshore intertidal walking

Inshore subtidal

Inshore subtidal

Inshore intertidal dive/snorkel

Inshore subtidal

Inshore subtidal

Inshore subtidal

Inshore subtidal

Inshore intertidal walking

Inshore subtidal

Inshore subtidal

Inshore subtidal

Inshore subtidal

Inshore subtidal

Inshore intertidal dive/snorkel

Inshore subtidal

Inshore subtidal

Inshore subtidal 


\begin{tabular}{|c|c|c|}
\hline Station \# & Locations & Bryce et al. 2018 (Figure 2) \\
\hline 68 & White Island & Inshore subtidal \\
\hline 69 & Outcrop NW Black Rocks & Inshore subtidal \\
\hline 74 & Beagle Reef & Inshore subtidal \\
\hline 75 & Beagle Reef & Inshore subtidal \\
\hline 77 & Mavis Reef & Inshore subtidal \\
\hline 78 & Mavis Reef & Inshore subtidal \\
\hline 79 & Albert Reef & Inshore subtidal \\
\hline 80 & Brue Reef & Inshore subtidal \\
\hline 83 & Brue Reef & Inshore subtidal \\
\hline 86 & King and Conway Islands & Inshore subtidal \\
\hline 88 & King and Conway Islands & Inshore subtidal \\
\hline 93 & White Island & Inshore subtidal \\
\hline 94 & De Freycinet Island & Inshore subtidal \\
\hline 95 & De Freycinet Island & Inshore subtidal \\
\hline 96 & Hedley Island & Inshore subtidal \\
\hline 99 & Outcrop North of Colbert Island & Inshore subtidal \\
\hline 101 & Browse Island & Midshelf subtidal \\
\hline 102 & Browse Island & Midshelf subtidal \\
\hline 105 & Browse Island & Midshelf subtidal \\
\hline 106 & Browse Island & Midshelf subtidal \\
\hline 110 & Jamieson Reef & Inshore subtidal \\
\hline 111 & Jamieson Reef & Inshore subtidal \\
\hline 113 & Condillac Island & Inshore subtidal \\
\hline 114 & Patricia Island & Inshore subtidal \\
\hline 115 & Heritage Reef & Inshore subtidal \\
\hline 116 & West Montalivet Island & Inshore subtidal \\
\hline 117 & West Montalivet Island & Inshore subtidal \\
\hline 118 & Robroy Reefs & Inshore subtidal \\
\hline 119 & Robroy Reefs & Inshore subtidal \\
\hline 136 & Ashmore Reef & Offshore subtidal lagoona \\
\hline 164 & Imperieuse Reef (Rowley Shoals) & Offshore subtidal lagoona \\
\hline
\end{tabular}

\section{C3: Inshore intertidal walking}

$$
\text { Fraser Island }
$$

Woodward Island Mermaid Reef (Rowley Shoals) Mermaid Reef (Rowley Shoals) Clerke Reef (Rowley Shoals) Clerke Reef (Rowley Shoals) Clerke Reef (Rowley Shoals) Clerke Reef (Rowley Shoals)
Inshore subtidal

Inshore intertidal walking

Offshore subtidal fore-reef slopes

Offshore subtidal lagoonal

Offshore subtidal fore-reef slopes

Offshore subtidal fore-reef slopes

Offshore subtidal lagoonal

Offshore subtidal fore-reef slopes 


\begin{tabular}{|c|c|c|}
\hline Station \# & Locations & Bryce et al. 2018 (Figure 2) \\
\hline 155 & Clerke Reef (Rowley Shoals) & Offshore subtidal lagoonal \\
\hline 156 & Clerke Reef (Rowley Shoals) & Offshore subtidal fore-reef slope \\
\hline 157 & Imperieuse Reef (Rowley Shoals) & Offshore subtidal fore-reef slopes \\
\hline 158 & Imperieuse Reef (Rowley Shoals) & Offshore subtidal fore-reef slope \\
\hline 159 & Imperieuse Reef (Rowley Shoals) & Offshore subtidal lagoonal \\
\hline 160 & Imperieuse Reef (Rowley Shoals) & Offshore intertidal walking \\
\hline 161 & Imperieuse Reef (Rowley Shoals) & Offshore subtidal fore-reef slope \\
\hline 162 & Imperieuse Reef (Rowley Shoals) & Offshore subtidal lagoonal \\
\hline 163 & Imperieuse Reef (Rowley Shoals) & Offshore intertidal walking \\
\hline 165 & Imperieuse Reef (Rowley Shoals) & Offshore subtidal fore-reef slope \\
\hline 166 & Imperieuse Reef (Rowley Shoals) & Offshore intertidal walking \\
\hline 167 & Imperieuse Reef (Rowley Shoals) & Offshore subtidal fore-reef slope \\
\hline 168 & Imperieuse Reef (Rowley Shoals) & Offshore subtidal fore-reef slope \\
\hline 169 & Clerke Reef (Rowley Shoals) & Offshore intertidal walking \\
\hline 170 & Clerke Reef (Rowley Shoals) & Offshore subtidal fore-reef slope \\
\hline 171 & Clerke Reef (Rowley Shoals) & Offshore subtidal lagoonal \\
\hline 172 & Clerke Reef (Rowley Shoals) & Offshore intertidal walking \\
\hline 173 & Clerke Reef (Rowley Shoals) & Offshore subtidal fore-reef slope \\
\hline 174 & Clerke Reef (Rowley Shoals) & Offshore intertidal walking \\
\hline 175 & Clerke Reef (Rowley Shoals) & Offshore subtidal lagoonal \\
\hline 177 & Mermaid Reef (Rowley Shoals) & Offshore intertidal walking \\
\hline 178 & Mermaid Reef (Rowley Shoals) & Offshore subtidal fore-reef slope \\
\hline 179 & Mermaid Reef (Rowley Shoals) & Offshore subtidal lagoonal \\
\hline 180 & Mermaid Reef (Rowley Shoals) & Offshore intertidal walking \\
\hline \multicolumn{3}{|c|}{ C4: Offshore intertidal dive/snorkel } \\
\hline 3 & Adele Island & Inshore intertidal walking \\
\hline 14 & Montgomery Reef & Inshore intertidal walking \\
\hline 15 & Montgomery Reef & Inshore intertidal walking \\
\hline 17 & Montgomery Reef & Inshore intertidal walking \\
\hline 19 & Montgomery Reef & Inshore intertidal walking \\
\hline 26 & Montgomery Reef & Inshore intertidal walking \\
\hline 33 & Cassini Island & Inshore intertidal dive/snorkel \\
\hline 35 & Cassini Island & Inshore intertidal dive/snorkel \\
\hline 37 & Cassini Island & Inshore intertidal dive/snorkel \\
\hline 38 & Cassini Island & Inshore intertidal dive/snorkel \\
\hline 39 & Cassini Island & Inshore subtidal \\
\hline 41 & Cassini Island & Inshore intertidal dive/snorkel \\
\hline 45 & Long Reef & Inshore intertidal walking \\
\hline 47 & Long Reef & Inshore subtidal \\
\hline 48 & Long Reef & Inshore intertidal walking \\
\hline
\end{tabular}




\begin{tabular}{|c|c|c|}
\hline Station \# & Locations & Bryce et al. 2018 (Figure 2) \\
\hline 50 & Long Reef & Inshore intertidal dive/snorkel \\
\hline 51 & Long Reef & Inshore intertidal walking \\
\hline 52 & Long Reef & Inshore intertidal walking \\
\hline 54 & Long Reef & Inshore intertidal dive/snorkel \\
\hline 55 & Long Reef & Inshore intertidal walking \\
\hline 56 & Long Reef & Inshore intertidal walking \\
\hline 59 & Cassini Island & Inshore intertidal walking \\
\hline 60 & Cassini Island & Inshore intertidal walking \\
\hline 61 & Wildcat Rocks & Inshore intertidal walking \\
\hline 62 & Champagney Islands & Inshore intertidal walking \\
\hline 65 & White Island & Inshore intertidal walking \\
\hline 66 & White Island & Inshore intertidal walking \\
\hline 76 & Mavis Reef & Inshore subtidal \\
\hline 81 & Brue Reef & Inshore intertidal walking \\
\hline 97 & Hedley Island & Inshore intertidal walking \\
\hline 98 & Hedley Island & Inshore intertidal walking \\
\hline 103 & Browse Island & Midshelf intertidal walking \\
\hline 112 & Condillac Island & Inshore intertidal walking \\
\hline 129 & Ashmore Reef & Offshore intertidal dive/snorkel \\
\hline 131 & Ashmore Reef & Offshore intertidal dive/snorkel \\
\hline 138 & Ashmore Reef & Offshore intertidal dive/snorkel \\
\hline 139 & Ashmore Reef & Offshore subtidal lagoonal \\
\hline 141 & Ashmore Reef & Offshore intertidal dive/snorkel \\
\hline \multicolumn{3}{|c|}{ C5: Inshore intertidal dive/snorkel } \\
\hline 122 & Ashmore Reef & Offshore subtidal lagoonal \\
\hline 124 & Ashmore Reef & Offshore subtidal lagoonal \\
\hline 125 & Ashmore Reef & Offshore subtidal fore-reef slopes \\
\hline 126 & Ashmore Reef & Offshore subtidal fore-reef slopes \\
\hline 127 & Ashmore Reef & Offshore subtidal fore-reef slopes \\
\hline 128 & Ashmore Reef & Offshore subtidal fore-reef slopes \\
\hline 130 & Ashmore Reef & Offshore subtidal fore-reef slopes \\
\hline 132 & Ashmore Reef & Offshore subtidal fore-reef slopes \\
\hline 133 & Ashmore Reef & Offshore subtidal fore-reef slopes \\
\hline 134 & Ashmore Reef & Offshore subtidal fore-reef slopes \\
\hline 135 & Ashmore Reef & Offshore subtidal fore-reef slopes \\
\hline 140 & Ashmore Reef & Offshore subtidal fore-reef slopes \\
\hline 142 & Hibernia Reef & Offshore subtidal fore-reef slopes \\
\hline 143 & Hibernia Reef & Offshore subtidal fore-reef slopes \\
\hline 144 & Hibernia Reef & Offshore subtidal fore-reef slopes \\
\hline 145 & Hibernia Reef & Offshore subtidal fore-reef slopes \\
\hline
\end{tabular}




\section{REFERENCES}

ABRS (2011). Australian Faunal Directory. Australian Biological Resources Study: Canberra, Australia; online at http://www.environment.gov.au/ biodiversity/abrs/online-resources/fauna/afd/ index.html. Accessed 2008-2016.

Alderslade, P. (1986). New species of the gorgonian genus Plumigorgia (Coelenterata: Octocorallia) with a review of the family Ifalukellidae. The Beagle, Occasional Papers of the Northern Territory Museum of Arts and Sciences 3: 92-122.

Alderslade, P. (2001). Six new genera and six new species of soft corals, and some proposed familial and subfamilial changes within Alcyonacea (Coelenterata: Octocorallia). Bulletin of the Biological Society of Washington 10: 15-65.

Baum, G., Januar, I., Ferse, S.C.A., Wild, C. and Kunzmann, A. (2016). Abundance and physiology of dominant soft coral linked to water quality in Jakarta Bay, Indonesia. PeerJ 4, e2625.

Bayer, F.M. (1981). Key to the genera of Octocorallia exclusive of Pennatulacea (Coelenterata: Anthozoa), with diagnoses of new taxa. Proceedings of the Biological Society of Washington 94: 901-947.

Benayahu, Y. (1985). Faunistic composition and patterns in the distribution of soft corals (Octocorallia: Alcyonacea) along the coral reefs of Sinai Peninsula. In: Gabrié, C. et al. (eds). Procedings of the $5^{\text {th }}$ International Coral Reef Congress. Vol. 6. Antenne Museum-EPHE, Moorea, French Polynesia, pp 255260.

Benayahu, Y. and Loya, Y. (1981). Competition for space among coral-reef sessile organisms at Eilat, Red Sea. Bulletin of Marine Science 31: 514-522.

Benayahu, Y. Shlagman, A. and Schleyer, M.H. (2003). Corals of the south-west Indian Ocean: VI. The Alcyonacea (Octocorallia) of Mozambique, with a discussion of soft coral distribution on south equatorial east African reefs. Zoologische Verhandelingen Leiden 345: 49-57.

Benayahu, Y., van Ofwegen, L.P., Dai, C-f., Jeng, M-S, Soong K., Shlagman, A., Hsieh, H.J. and McFadden, C.S. (2012). Diversity, distribution, and molecular systematics of octocorals (Coelenterata: Anthozoa) of the Penghu Archipelago, Taiwan. Zoological Studies 51(8): 1529-1548.

Blakeway, D. and Radford, B.T.M. (2004). Scleractinian Corals of the Dampier Port and inner Mermaid Sound: species list, community composition and distributional data. MScience, 2005: 1-11.

Bradbury, R.H. and Mundy, C. (1989). Large-scale shift in biomass of the Great Barrier Reef ecosystem. In: Sherman, K. and Alexander, L.M. (eds). Biomass yields and geography of large marine ecosystems. Westview Press, Washington D.C.: 143-167.

Broch, H. (1916). Results of Dr. E. Mjöberg's Swedish scientific expedition to Australia 1910-1913. XI. Alcyonarien. Kunglia Svenska Vetenskapsakademiens Handlingar 52(11): 1-52.
Breedy, O., van Ofwegen, L.P. and Vargas S. (2012). A new family of soft corals revealed by integrative taxonomy. Systematics and Biodiversity 10: 351-359.

Bryce, C. (ed.) (2009). Marine biodiversity survey of Mermaid Reef (Rowley Shoals), Scott and Seringapatam Reef. Records of the Western Australian Museum Supplement 77.

Bryce, C., Bryce, M. and Radford, B. (2018). Project methods and station geomorphology related to a multi-taxon survey (2009-2014) of the Kimberley. Records of the Western Australian Museum Supplement 85: 1-43. doi: 10.18195/issn.0313-122x.85.2018.001-043

Bryce, M. and Sampey, A. (2014). Kimberley marine biota. Historical data: soft corals and sea fans (Octocorallia). Records of the Western Australian Museum Supplement 84: 159-168. doi: 10.18195/ issn.0313-122x.84.2014.059-168

Bryce, M. and Poliseno, A. (2014). Two new records of octocorals (Anthozoa, Octocorallia) from north-west Australia. Records of the Western Australian Museum 29: 101-110.

Bryce, M., Poliseno, A. Alderslade, P. and Vargas S. (2015). Digitate and capitate soft corals (Cnidaria: Octocorallia: Alcyoniidae) from Western Australia with reports on new species and new Australian geographical records. Zootaxa 3963: 160-200.

Chanmethakul, T., Chansang, H. and Watanasit, S. (2010). Soft coral (Cnidaria: Alcyonacea) distribution patterns in Thai waters. Zoological Studies 49(1): 72-84.

Clarke, K.R. and Warwick, R.M. (2001). Changes in marine communities: An approach to statistical analysis and interpretations, 2nd edition. PRIMER-E, Plymouth.

Clarke, K.R. and Gorley, R.N. (2006). PRIMER v6 user manual. PRIMER-E, Plymouth.

De Cáceres, M. and Legendre, P. (2009). Associations between species and groups of sites: indices and statistical inference. Ecology 90(12): 3566-3574.

De Cáceres, M., Legendre, P., Moretti, M. (2010). Improving indicator species analysis by combining groups of sites. Oikos 119(10): 1674-1684.

Department of Environment and Conservation (2009). Protecting the Kimberley. A synthesis of scientific knowledge to support conservation management in the Kimberley region of Western Australia: Perth.

Department of Parks and Wildlife (2016). North Kimberley Marine Park Joint management plan 2016 Uunguu, Balanggarra, Miriuwung Gajerrong, and Wilinggin management areas, Number plan 89 Department of Parks and Wildlife, Perth, pp. 64.

Dufrêne, M. and Legendre, P. (1997). Species assemblages and indicator species: The need for a flexible asymmetrical approach. Ecological Monographs 67: 345-366.

Edmunds, P.J., Adjeroud, M., Baskett, M.L., Baums, B., Budd, A.F., Carpenter, R.C., Fabina, N., Fan, T.-Y., Franklin, E.C., Gross, K., Han, X., Jacobson, L., Klaus, J.S., McClanaham, T.R., O'Leary. J.K., van Oppen, M.J.H., Pochon, X., Putnam, H.M., Smith, T.B., Stat, M.I., Sweatman, R., van Woesik, R. and Gates, R.D. (2014). Persistence and change in community composition of reef corals through past, present, and future climates. PLoS One 9: e107525. 
EPA (2004). Benthic primary producer habitat protection for Western Australia's Marine Environment. Draft guidance No. 29. Environmental Protection Authority, Perth Australia.

Fabricius, K. (1996) Ecosystem recovery after episodic disturbance: resilience of some coral reefs after Acanthaster outbreaks. Senckenbergiana maritima 27: 227-235

Fabricius, K. (1997) Soft coral abundance in the central Great Barrier Reef: Effects of Acanthaster planci, space availability and aspects of the physical environment. Coral Reefs 16: 159-167.

Fabricius, K. (1998) Reef invasion by soft corals: Which taxa and which habitats? In: Greenwood, J.G and Hall, N.J. (eds.) Proceedings of the Australian Coral Reef Society 75th Anniversary Conference, Heron Island, October 1997. School of Marine Science, University of Queensland, Brisbane, pp. 77-90.

Fabricius, K. (2008). A brief photo guide to the shallow-water octocorals of the Rowley Shoals, Western Australia. Report to the Department of Environment and Conservation, Government of Western Australia: 1-39.

Fabricius, K. and Alderslade, P. (2001). Soft corals and sea fans: a comprehensive guide to the tropical shallow water genera of the central-west Pacific, the Indian Ocean and the Red Sea. Australian Institute of Marine Science: Townsville, Queensland.

Fabricius, K. and Déath, G. (2001). Biodiversity of the Great Barrier Reef: large-scale patterns and turbidityrelated local loss of soft coral taxa. In: E. Wolanski (ed.) Oceanographic processes of coral reefs: physical and biological links in the Great Barrier Reef. Boca Raton, FL: CRC Press, pp. 127-144.

Fabricius, K.E., Alderslade, P., Williams, G.C., Colin, P.L. and Golbuu, Y. (2007). Octocorallia in Palau, Micronesia: Effects of biogeography and coatal influences on local and regional biodiversity Chapter 7: 70-92. In: Kayanne, H., Omori, M., Fabricius, K. Verheij, E. Colin, P. Golbuu, Y. and Yurihira H (eds) (2007). Coral Reefs of Palau. Palau International Coral Reef Centre, Palau.

Fromont, J., Wahab, M.A.A., Gomez, O. Ekins, M. Grol, M. and Hooper, J.N.A. (2016). Patterns of sponge biodiversity in the Pilbara, Northwestern Australia. Diversity, 8(21): 1-22. doi: 10.3390/d8040021

Fox, H.E., Dahuri, R. and Cladwell R.L. (2003). Recovery in rubble fields: long-term impacts of blast fishing. Marine Pollution Bulletin 46: 1024-1031.

Grasshoff, M. and Bargibant, G. (2001). Les gorgones des recifs coralliens de Nouvelle-Caledonie: 1-335. IRD, New Caledonia.

Griffith, J. (1997). The corals collected during September/ October 1997 at Ashmore Reef, Timor Sea. Unpublished report to Parks Australia.

Heyward, A., Jones, R., Meeuwig J., Burns, K., Radford, B., Colquhoun, J., Cappo, M., Case, M., O'Leary R., Fisher, R., Meekan, M. and Stower, M. (2012). Montara: 2011 Offshore Banks Assessment Survey. Final report prepared by the Australian Institute of Marine Science for PTTEP Australasia (Ashmore Cartier) Pty. Ltd. In accordance with contract no. 000/2011/02-04, pp. 253.
Hoegh-Guldberg, O., Mumby, P.J., Hooten, A.J., Steneck, R.S., Greenfield, P., Gomez, E., Harvell, C.D., Sale, P.F., Edwards, A.J., Caldeira, K., Knowlton, N., Eakin, C.M., Iglesias-Prieto, R., Muthiga, N., Bradbury, R.H., Dubi, A. and Hatziolos, M.E. (2007). Coral reefs under rapid climate change and ocean acidification. Science 318: 1737-1742.

Hooper N.A.J. and Ekins M. (2004). Collation and validation of museum collection databases related to the distribution of marine sponges in northern Australia. Technical reports of Queensland Museum 2: 1-90.

Hughes, T.P., Graham, N.A.J., Jackson, J.B.C., Mumby, P.J. and Steneck, R.S. (2010). Rising to the challenge of sustaining coral reef resilience. Trends in Ecology and Evolution 25: 633-642.

Janes M.P. and Mary A.G. (2012). Synopsis of the family Xeniidae (Cnidaria: Octocorallia): Status and Trends. Proceedings of the $12^{\text {th }}$ International Coral Reef Symposium, Cairns, Australia, 9-13 July 2012, pp. 5.

Jeng, M.S., Huang, H.D., Dai, C.F., Hsiao, Y.C. and Benayahu, Y. (2011). Sclerite calcification and reefbuilding in the fleshy octocoral genus Sinularia (Octocorallia: Alcyonacea). Coral Reefs 30: 925-933.

Keesing, J.K., Irvine, T.R., Alderslade, P., Clapin, G., Fromont, J., Hosie, A.M., Huisman, J.M., Phillips, J.C., Naughton, K.M., Marsh, L.M., Slack-Smith, S.M., Thomson, D.P. and Watson, J.E. (2011). Marine benthic flora and fauna of Gourdon Bay and the Dampier Peninsula in the Kimberley region of north-western Australia. Journal of the Royal Society of Western Australia 94: 285-301.

Kükenthal, W. (1910). Die Fauna Südwest-Australiens. Ergebnisse der Hamburger südwest-australischen Forschungsreise 1905. Gustav Fisher-Verlag, Jena.

Lenz, E.A.L., Bramanti, L., Lasker, H.R. and Edmunds P.J. (2015). Long-term variation in the population density of gorgonians in St. John, US Virgin Islands. Coral Reefs. doi: 10.1007/s00338-015-1315-x.

López-González P.J., Grinyó J. and Gili J-M. (2014). Chironephthya mediterranean. sp. (Octocorallia, Alcyonacea, Nidaliidae), the first species of the genus discovered in the Mediterranean Sea. Marine Biodiversity 45(4): 667-688. doi: 10.1007/s12526-0140269-5.

Marsh, L.M. (1986). Part III: Cnidaria, other than reef-building corals (pp. 37-38). In: Berry, PF (ed.), Faunal Surveys of the Rowley Shoals and Seringapatam Reef, North-western Australia. Records of the Western Australian Museum Supplement 25. Western Australian Museum: Perth.

Marsh, L.M. (1992). Part II. Scleractinian and other hard corals (pp. 15-22). In: Morgan, G.J. (ed.), Survey of the Aquatic Fauna of the Kimberley Islands and Reefs, Western Australia, Western Australian Museum: Perth.

Marsh, L.M. (1993). Cnidaria, other than reef-building corals, of Ashmore Reef and Cartier Island (pp. 21-22). In: Berry, P.F. (ed.), Marine faunal surveys of Ashmore Reef and Cartier Island. Records of the Western Australian Museum Supplement 44. Western Australian Museum: Perth. 
Masini, R, Sim, C.B. and Simpson, C.J. (2009). Protecting the Kimberley. A synthesis of scientific knowledge to support conservation management in the Kimberley region of Western Australia. Department of Environment and Conservation, Western Australia.

McFadden, C.S., van Ofwegen, L.P., Beckman, E., Benayahu, Y. and Alderslade, P. (2013). Molecular systematics of the speciose Indo-Pacific soft coral genus, Sinularia (Anthozoa: Octocorallia). Invertebrate Biology 128: 303-323.

McFadden, C.S. and van Ofwegen, L.P. (2013). Molecular phylogenetic evidence supports a new family of octocorals and a new genus of Alcyoniidae (Octocorallia, Alcyonacea). ZooKeys 346: 59-83.

McFadden, C.S., Haverkort-Yeh, R., Reynolds, A.M., Halàsz, A., Quattrini, A.M., Forsman, Z.H., Benayahu, Y. and Toonen, R.J. (2017). Species boundaries in the absence of morphological, ecological or geographical differentiation in the Red Sea octocoral genus Ovabunda (Alcyonacea: Xeniidae). Molecular Phylogenetics and Evolution 112: 174-184.

Moore, C.H., Radford, B.T., Possingham, H.P., Andrew J., Heyward, A.J., Stewart, R.R., Watts, M.E., Prescott, J., Newman, S.J., Harvey, E.S., Fisher, R., Bryce, C.W., Lowe, R.J., Berry, O., Espinosa-Gayosso, A., Sporer, E. and Saunders, T. (2016). Improving spatial prioritisation for remote marine regions: optimising biodiversity conservation and sustainable development trade-offs. Nature Scientific Reports 6: 32029. doi: 10.1038/srep32029

Pitcher, C.R., Miller, M., Morello, E., Fry, G., Strzelecki, J., McLeod, I., Slawinski, D., Ellis, N., Thomson, D., Bearham, D.,Keesing, J., Donovan, A., Mortimer, N. Babcock, R., Fromont, J, Gomez, O., Hosie, A., Hara, A., Moore, G., Morrison, S., Kirkendale, L., Whisson, C., Richards, Z., Bryce, M., Marsh, L., Naughton, K., O'Loughlin, M., O'Hara, T., Boddington, D. and Huisman, J. (2016). Environmental Pressures: Regional Biodiversity - Pilbara Seabed Biodiversity. Mapping and Characterisation. Final report, CSIRO Oceans and Atmosphere, Published Brisbane, March 2016.

Przeslawski, R. Ahyong, S., Byrne, M, Wörheide, G. Hutchins, P. (2008). Beyond corals and fish: The effects of climate change on noncoral benthic invertebrates of tropical reefs. Global Change Biology 14: 2773-2795.

Pyke, G. H. and Ehrlich, P. R. (2010). Biological collections and ecological/environmental research: a review, some observations and a look to the future. Biological Reviews 85(2): 247-266.
Richards, Z.T., Bryce, M. and Bryce, C. (2013). New records of atypical coral reef habitat in the Kimberley, Australia. Journal of Marine Biology, Article ID 363894: 1-8. doi: 10.1155/2013/363894.

Richards, Z.T., Bryce, M., Bryce, C. (2018). The composition and structure of shallow benthic reef communities in the Kimberley, NW Australia. Records of the Western Australian Museum Supplement 85: Submitted.

Ruzicka, R.R., Colella, M.A., Porter, J.W., Morrison J.M., Kidney, J.A., Brinkhuis, V., Lunz, K.A., Macaulay, K.A., Bartlett, L.A., Meyers, M.K. and Colee, J. (2013). Temporal changes in benthic assemblages on Florida Keys reefs 11 years after the 1997/1998 El Niño. Marine Ecology Progress Series 489: 125-141.

Sampey, A., Bryce, C., Osborne, S. and Miles, A. (2014). Kimberley marine biota: Historical data: introduction and methods. Records of the Western Australian Museum Supplement 84: 19-43. doi: 10.18195/issn.0313122x.84.2014.019-043

Shackleton, M. and Rees, G.N. (2016). DNA barcoding Australian macroinvertebrates for monitoring programs: benefits and current shortcomings. Marine and Freshwater Research 67: 380-390.

Smith, J.E., Brainard, R., Carter, A., Grillo, S., Edwards, C., Harris, J., Lewis, L. Obura, D., Rohwer, F., Sala, E., Vroom, P.S. and Sandin, S. (2016). Re-evaluating the health of coral reef communities: baselines and evidence for human impacts across the central Pacific. Proceedings of the Royal Society B 283: 20151985. doi: 10.1098/rspb. 2015.1985

Tursch, B. and Tursch, A. (1982). The soft coral community on a sheltered reef quadrat at Laing Island (Papua New Guinea). Marine Biology 68: 321-332.

Williams, G.C. (1992). Biogeography of the octocorallian coelenterate fauna of southern Africa. Biological Journal of the Linnean Society 46: 351-401.

Wilson, B. (2013). The Biogeography of the Australian North West Shelf: Environmental change and life's response. Elsevier: Burlington, Massachusetts.

Wilson, B. (2014). Kimberley marine biota. History and Environment. Records of the Western Australian Museum Supplement 84: 1-18. doi: 10.18195/ issn.0313-122x.84.2014.001-018

Wood, M. and Mills, D. (2008). A Turning of the tide: science for decisions in the Kimberley-Browse marine region. Western Australian Marine Science Institute: Perth. 